\title{
Stability of cosmological deflagration fronts
}

\author{
Ariel Mégevand* and Federico Agustín Membield† \\ IFIMAR (CONICET-UNMdP) and \\ Departamento de Física, Facultad de Ciencias Exactas y Naturales, \\ UNMdP, Deán Funes 3350, (7600) Mar del Plata, Argentina
}

\begin{abstract}
In a cosmological first-order phase transition, bubbles of the stable phase nucleate and expand in the supercooled metastable phase. In many cases, the growth of bubbles reaches a stationary state, with bubble walls propagating as detonations or deflagrations. However, these hydrodynamical solutions may be unstable under corrugation of the interface. Such instability may drastically alter some of the cosmological consequences of the phase transition. Here, we study the hydrodynamical stability of deflagration fronts. We improve upon previous studies by making a more careful and detailed analysis. In particular, we take into account the fact that the equation of motion for the phase interface depends separately on the temperature and fluid velocity on each side of the wall. Fluid variables on each side of the wall are similar for weakly first-order phase transitions, but differ significantly for stronger phase transitions. As a consequence, we find that, for large enough supercooling, any subsonic wall velocity becomes unstable. Moreover, as the velocity approaches the speed of sound, perturbations become unstable on all wavelengths. For smaller supercooling and small wall velocities, our results agree with those of previous works. Essentially, perturbations on large wavelengths are unstable, unless the wall velocity is higher than a critical value. We also find a previously unobserved range of marginally unstable wavelengths. We analyze the dynamical relevance of the instabilities, and we estimate the characteristic time and length scales associated to their growth. We discuss the implications for the electroweak phase transition and its cosmological consequences.
\end{abstract}

\section{INTRODUCTION}

The development of a cosmological first-order phase transition via nucleation and expansion of bubbles provides an interesting scenario for the formation of cosmological objects such as magnetic fields [1], topological defects [2], baryon inhomogeneities [3 5], gravitational waves [6, 7], or the baryon asymmetry of the universe [8]. One of the relevant aspects of the dynamics of a first-order phase transition is the motion of the transition fronts. In most cases, stationary solutions exist, and the bubble growth reaches a terminal velocity shortly after the bubble nucleates. The velocity of bubble walls depends on the friction with the plasma [9, 10] and on hydrodynamics [11]. Thus, for a given set of parameters, there can be one or more solutions. For instance, the wall may propagate as a supersonic detonation

\footnotetext{
*Member of CONICET, Argentina; megevand@mdp.edu.ar

${ }^{\dagger}$ Fellow of CONICET, Argentina; membiela@mdp.edu.ar
} 
or as a subsonic deflagration. The cosmological consequences of a phase transition depend strongly on the wall velocity. For example, detonations favor the generation of gravitational waves, whereas weak deflagrations favor electroweak baryogenesis. It is well known that these hydrodynamic solutions may be unstable [12 14, 16, 17]. Such instabilities would have important implications for the cosmological remnants of the phase transition.

The standard approach to the stability of combustion or phase transition fronts is to consider small perturbations of the wall surface and the fluid [12]. In the cosmological context, the stability of deflagrations was first studied in Ref. [13], in the non-relativistic limit. This analysis was improved in Ref. [14] by considering relativistic velocities, and by taking into account the dependence of the velocity of phase transition fronts on temperature. The latter is the most important difference with previous analysis, since temperature fluctuations cause velocity fluctuations which may stabilize the wall. Numerical simulations [15] agree with this stabilization.

A very simple expression for the wall velocity was considered in Ref. [14], namely, $v_{w} \propto$ $T_{c}-T_{+}$, where $T_{c}$ is the critical temperature and $T_{+}$is the temperature outside the bubble. Perturbing this equation gives an equation for the perturbations of the interface, which involves the temperature fluctuations $\delta T_{+}$. We wish to point out, however, that such a simple form of the wall velocity does not take into account (among other things) the dependence on temperature perturbations $\delta T_{-}$behind the wall (which were otherwise considered in [14] as independent from $\delta T_{+}$). Furthermore, the results were applied to a specific case (the electroweak phase transition) without taking into account the fact that the temperature $T_{+}$ is higher than the nucleation temperature $T_{N}$, due to reheating in front of the wall.

These issues are not relevant in the case of small wall velocity, small latent heat, and little supercooling, but can be important otherwise. The discussion on the electroweak phase transition in Ref. [14] was carried out for the minimal standard model (SM) with a Higgs mass $m_{H}=40 \mathrm{GeV}$. This gives a rather weak phase transition (although strong enough for baryogenesis), for which the aforementioned approximations are valid. The deflagration was found to be stable for wall velocities above a critical value $v_{c}=0.07$. Thus, arguing that the wall velocity is generally $v_{w} \gtrsim 0.1$, it was concluded that the deflagration is commonly stable. However, the wall velocity depends on the details of the specific model, and may be smaller. Furthermore, many extensions of the SM give stronger phase transitions, which would give higher values of $v_{c}$.

In this paper we aim to perform a more complete calculation, and to investigate a wider range of parameters. The main improvements of our treatment will be to consider a more realistic equation for the wall velocity, which depends on the fluid fluctuations on both sides of the interface, and to take into account the effects of reheating, i.e., the fact that the temperature $T_{+}$in front of the wall is not a boundary condition for the deflagration. In the limit of small wall velocities, little supercooling, and small latent heat, our results agree with those of Ref. [14]. Increasing the latent heat or the wall velocity does not change the qualitative picture. However, as the amount of supercooling is increased, the critical velocity increases much more quickly than what found in Ref. [14], even though the reheating effect tends to stabilize the deflagration. As a consequence, for strong enough supercooling the wall propagation is unstable for any velocity below the speed of sound. moreover, the instability is stronger at the speed of sound. This is in contradiction with the results of Ref. [14]. The origin of the discrepancy is in the fact that, in our treatment, the wall velocity depends on fluid fluctuations on both sides of the wall.

The paper is organized as follows. In the next section we review the stationary motion 
of a phase transition front. In Sec. III we consider linear perturbations of the interface and the fluid. We discuss the approaches and results of previous works, and then we derive the equations for the perturbations and find the general solution. In Sec. IV we study the possible instabilities of the deflagration and compare our results with previous works. We find analytical approximations for the case of small velocity, small latent heat, and little supercooling. We also discuss how the reheating which occurs in front of the wall affects the stability of a deflagration. In Sec. $\mathrm{V}$ we use the bag equation of state to study the instability as a function of the relevant parameters. We explore numerically a wide region of parameter space. In Sec. VI we consider the dynamics of the instabilities in a cosmological phase transition, and in Sec. VII we discuss the results for the specific case of the electroweak phase transition. We also discuss briefly on some cosmological effects. Finally, in Sec. VIII we summarize our conclusions.

\section{PHASE TRANSITION DYNAMICS AND STATIONARY WALL MOTION}

Cosmological phase transitions are generally a consequence of the high temperature behaviour of a theory with spontaneous symmetry breaking. Macroscopically, the system can be described by a relativistic fluid and a scalar field $\phi$ which acts as an order parameter. The free energy density $\mathcal{F}(\phi, T)$ has different minima $\phi_{+}$and $\phi_{-}$at high and low temperatures, respectively. These minima characterize two different phases. For instance, in the case of the electroweak phase transition, $\phi$ corresponds to the expectation value of the Higgs field, and we have $\phi_{+}=0, \phi_{-} \sim T_{c} \sim 100 \mathrm{GeV}$.

If the phase transition is first-order, there is a range of temperatures at which these two minima coexist separated by a barrier. Thus, the metastable phase is characterized by the free energy density $\mathcal{F}_{+}(T)=\mathcal{F}\left(\phi_{+}, T\right)$, whereas the stable phase is characterized by $\mathcal{F}_{-}(T)=\mathcal{F}\left(\phi_{-}, T\right)$. The pressure in each phase is given by $p_{ \pm}=-\mathcal{F}_{ \pm}$, the entropy density by $s_{ \pm}=d p_{ \pm} / d T$, and the energy density by $e_{ \pm}=T s_{ \pm}-p_{ \pm}$. The critical temperature $T_{c}$ is defined by $\mathcal{F}_{+}\left(T_{c}\right)=\mathcal{F}_{-}\left(T_{c}\right)$. The latent heat is defined as the energy discontinuity at $T=$ $T_{c}$, and is given by $L=T_{c}\left[\mathcal{F}_{-}^{\prime}\left(T_{c}\right)-\mathcal{F}_{+}^{\prime}\left(T_{c}\right)\right]$. A first-order phase transition is characterized by the supercooling of the system (which remains in the metastable phase below $T_{c}$ ), followed by the nucleation and growth of bubbles of the stable phase at a temperature $T_{N}<T_{c}$ (see, e.g., [18-20]). The latent heat is released at the phase transition fronts, which are the bubble walls.

We are interested in the motion of the latter. Therefore, we shall consider the hydrodynamics of two phases separated by a moving interface. The equations for the wall and the fluid variables can be derived from the conservation of the stress tensor for the scalar field and the fluid. These can be written in the form

$$
\begin{array}{r}
\partial_{\mu}\left(-T \frac{\partial \mathcal{F}}{\partial T} u^{\mu} u^{\nu}+g^{\mu \nu} \mathcal{F}\right)+\partial_{\mu} \partial^{\mu} \phi \partial^{\nu} \phi=0 \\
\partial_{\mu} \partial^{\mu} \phi+\frac{\partial \mathcal{F}}{\partial \phi}+\tilde{\eta} T_{c} u^{\mu} \partial_{\mu} \phi=0
\end{array}
$$

with $u^{\mu}=(\gamma, \gamma \mathbf{v})$ the four velocity of the fluid and $g^{\mu \nu}$ the Minkowsky metric tensor. The terms in parenthesis in Eq. (1) give the well known stress tensor of a relativistic fluid

$$
T^{\mu \nu}=w u^{\mu} u^{\nu}-p g^{\mu \nu}
$$


where $w$ is the enthalpy and $p$ the pressure. The last term in Eq. (11) gives the transfer of energy between the plasma and the field. The motion of the latter is governed by Eq. (21). The last term in this equation is a phenomenological damping term ${ }^{1}$. The dimensionless coefficient $\tilde{\eta}$ can be obtained from microphysics calculations (in the general case, $\tilde{\eta}$ may depend on the field $\phi$ ).

For the macroscopic treatment, it is a good approximation to consider an infinitely thin interface. The system of equations (1) then gives the fluid equations on either of the phases (where the field is constant), as well as the connection between the solutions at each side of the interface. On the other hand, Eq. (2) gives an equation for the interface itself and the forces acting on it. Due to the friction with the surrounding plasma, the bubble walls in general reach a terminal velocity ${ }^{2}$. We shall now consider the stationary motion. In the next section we shall study perturbations of the stationary solutions. For simplicity, we shall assume a planar interface moving towards the positive $z$ axis.

\section{A. Fluid equations}

For planar symmetry, the problem becomes $(1+1)$-dimensional, and we need only consider the $z$ component of the fluid velocity, which we shall denote $v(z, t)$. Within each phase the field is a constant, and Eq. (11) just gives the conservation of energy and momentum of the fluid, $\partial_{\mu} T^{\mu \nu}=0$, with $T^{\mu \nu}$ given by Eq. (3). The absence of a distance scale in these equations justifies to assume the similarity condition, namely, that quantities depend only on the variable $\xi=z / t$. Thus, we have $\partial_{t}=-(\xi / t) d / d \xi$ and $\partial_{z}=(1 / t) d / d \xi$. Furthermore, variations of thermodynamical quantities are related by the speed of sound $c_{s}^{2}=d p / d e$. We have, e.g.,

$$
d p=d w /\left(1+c_{s}^{-2}\right) .
$$

We may thus obtain an equation for $v(\xi)$ which depends only on the parameter $c_{s}$ [12] $\left(c_{s}\right.$ depends on the equation of state (EOS) and will be in general a function of temperature). In the planar case this equation is very simple (see e.g. [26]). The solutions are either $v=$ constant, or the particular solution $v_{\text {rar }}(\xi)=\left(\xi-c_{s}\right) /\left(1-\xi c_{s}\right)$. The latter corresponds to a rarefaction wave. In this paper we shall be interested in the constant velocity solutions. For these, the temperature is also a constant.

\section{B. Matching conditions}

The fluid solutions on each side of the bubble wall can be linked by integrating Eq. (1) across the wall. It is convenient to consider a reference frame moving with the wall, where all time derivatives vanish. We obtain two equations, $\partial_{z} T^{z 0}=0, \partial_{z} T^{z z}=0$, and the integration

\footnotetext{
${ }^{1}$ Recently [21 23], different forms of the damping term have been proposed in order to account for the saturation of the friction force at ultra-relativistic velocities 24|. Since we shall only deal with deflagrations, the damping term in Eq. (2) is a good approximation [23, 25].

${ }^{2}$ In Ref. 24], it was shown that, if the wall reaches ultra-relativistic velocities, it may enter a stage of continuous acceleration. However, models which allow such ultra-relativistic velocities will not allow, in general, deflagrations. We are not interested in such models.
} 
gives simply

$$
\begin{aligned}
w_{-} v_{-} \gamma_{-}^{2} & =w_{+} v_{+} \gamma_{+}^{2}, \\
w_{-} v_{-}^{2} \gamma_{-}^{2}+p_{-} & =w_{+} v_{+}^{2} \gamma_{+}^{2}+p_{+},
\end{aligned}
$$

where + and - signs refer to variables just in front and just behind the wall, respectively. Notice that in this frame the fluid velocity is negative (Fig. 11).

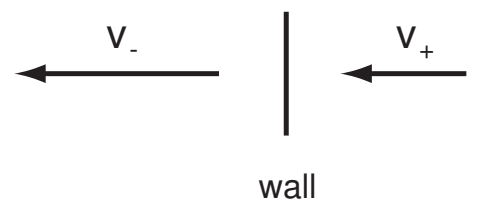

FIG. 1: Sketch of a deflagration in the wall frame.

These equations have two branches of solutions, called detonations and deflagrations. Detonations are characterized by the relation $\left|v_{+}\right|>\left|v_{-}\right|$, whereas deflagrations are characterized by $\left|v_{+}\right|<\left|v_{-}\right|$. For detonations, the curve of $\left|v_{+}\right|$vs $\left|v_{-}\right|$has a minimum at the Jouguet point $\left|v_{-}\right|=c_{s-}$, where $\left|v_{+}\right|$takes a value $v_{J}^{\text {det }}>c_{s+}$ (hence, for detonations the incoming flow is supersonic). For deflagrations, the curve of $\left|v_{+}\right|$vs $\left|v_{-}\right|$has a maximum value $v_{J}^{\text {def }}$ at the Jouguet point $c_{s-}$. In this case, $v_{+}$is subsonic, $v_{J}^{\text {def }}<c_{s+}$. Detonations are called weak if $v_{-}$is supersonic as well as $v_{+}$, and deflagrations are called weak if $v_{-}$is subsonic as well as $v_{+}$. If one of the velocities is supersonic and the other one subsonic, then the hydrodynamic process is called strong.

\section{Fluid profiles}

The profiles of the fluid velocity and temperature must be constructed from the solutions of the fluid equations on each side of the wall, using the matching conditions at the wall and the boundary conditions. The latter correspond to vanishing fluid velocity far behind and far in front of the wall. The value of the temperature $T_{N}$ far in front of the wall is also a boundary condition. We shall now describe briefly the possible fluid profiles. For details, see e.g. [26].

Let us call $\tilde{v}_{+}$and $\tilde{v}_{-}$the values of the fluid velocity on each side of the phase discontinuity. For a detonation, the fluid velocity $\tilde{v}_{+}$vanishes in front of the wall, which moves supersonically, i.e., we have $v_{w}=\left|v_{+}\right| \geq v_{J}^{\text {det }}>c_{s+}$. Behind the wall, we have a nonvanishing velocity $\tilde{v}_{-}$, and the wall is followed by a rarefaction wave. It turns out that only weak detonations can fulfil the boundary conditions. Therefore, strong detonations are not possible.

For a subsonic wall, the rarefaction solution $v_{\text {rar }}(\xi)$ cannot be accommodated in the velocity profile. The fluid velocity vanishes behind the wall $\left(\tilde{v}_{-}=0\right)$, and the hydrodynamic process is a weak deflagration, with $\left|v_{-}\right|=v_{w}<c_{s-}$. In front of the wall, the velocity is a constant up to a certain point where the velocity vanishes abruptly (see Fig. 2). Such a discontinuity without change of phase is called a shock front. At the shock discontinuity, Eqs. (5.66) still apply, but now the enthalpy and pressure are related by the same EOS on both sides of the interface. These equations give the temperature $T_{+}$as a function of the boundary condition $T_{N}$, as well as the velocity of the shock front. 


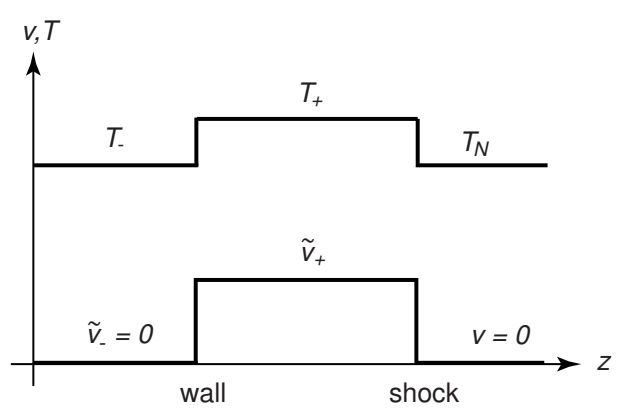

FIG. 2: Sketch of fluid velocity and temperature profiles for a deflagration, in the reference frame of the bubble center.

Between weak deflagrations and detonations there is a velocity gap $c_{s-}<v_{w}<v_{J}^{\text {det }}$. The "traditional" weak deflagration profile described above is in principle possible for a supersonic wall as well. In such a case, the hydrodynamic solution is a strong deflagration, with $\left|v_{-}\right|=v_{w}>c_{s_{-}}$. Numerical calculations [27] suggest that such a strong deflagration is unstable since, if set as initial condition, it evolves to other stationary solutions.

A supersonic deflagration can also be constructed using the solution $v_{\text {rar }}$ behind the wall (see Fig. 3). Instead of $\left|v_{-}\right|=v_{w}$, in this case the matching conditions only require $\left|v_{-}\right| \geq c_{s_{-}}$ (for details, see, e.g., [26]). Therefore, this kind of solution is either a strong or a Jouguet deflagration. In either case, since the fluid velocity $\tilde{v}_{-}$does not vanish, the wall velocity is given by the relativistic sum of $\left|v_{-}\right|$and $\tilde{v}_{-}$, and is always supersonic. In order to avoid a strong deflagration, the only possibility is a Jouguet solution, $v_{-}=-c_{s-}$. As shown in Fig. 3 (right panel), in this case the rarefaction begins immediately at the wall. Thus, the strong deflagration shown in the left panel is an intermediate case between the traditional strong deflagration of Fig. 2 and the supersonic Jouguet deflagration.

Notice that we have a family of solutions depending on two free parameters, namely $v_{-}$ and $\tilde{v}_{-}$. For $v_{-}=-v_{w}$ we obtain a reduced family of strong deflagrations, namely, those with the traditional profile, whereas for $v_{-}=-c_{s}$ we obtain the family of supersonic Jouguet deflagrations. Fixing the latter condition and varying the value of $\tilde{v}_{-}$, the velocity of the Jouguet deflagration fills the range of $v_{w}$ between the weak deflagration and the detonation.
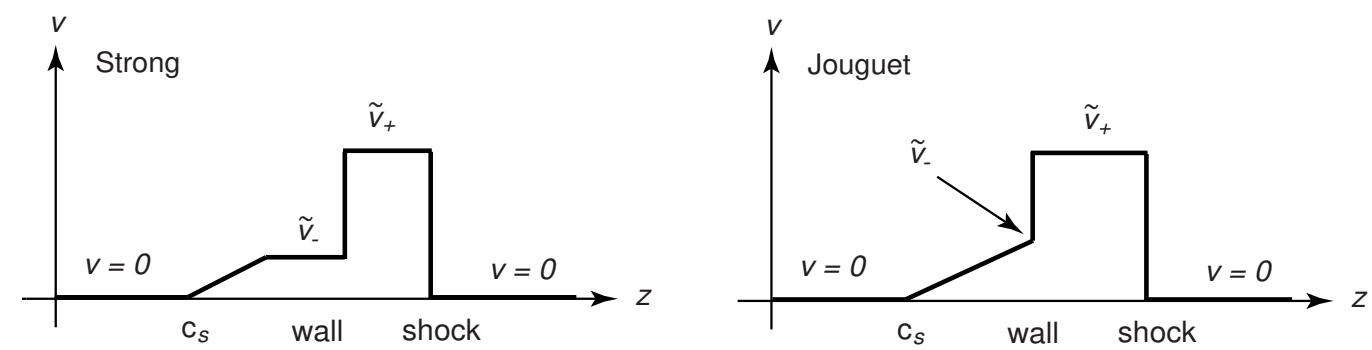

FIG. 3: Sketch of fluid velocity profile for strong and Jouguet deflagrations. 


\section{Equation for the interface}

We shall now obtain an equation for the interface from Eq. (2). In the reference frame of the wall, we multiply by $\phi^{\prime} \equiv d \phi / d z$ and then integrate across the interface. We obtain

$$
p_{-}-p_{+}+\int_{-}^{+}\left(-\frac{\partial \mathcal{F}}{\partial T}\right) \frac{d T}{d z} d z+\tilde{\eta} T_{c} \int_{-}^{+} \phi^{\prime 2} \gamma v d z=0
$$

where we have used the relation $(\partial \mathcal{F} / \partial \phi) d \phi / d z=d \mathcal{F} / d z-(\partial \mathcal{F} / \partial T) d T / d z$. In Eq. (7) we identify the force which drives the bubble expansion,

$$
F_{\mathrm{dr}}=p_{-}\left(T_{-}\right)-p_{+}\left(T_{+}\right)+\int_{-}^{+} \frac{\partial \mathcal{F}}{\partial T} \frac{d T}{d z} d z
$$

and the friction force, which explicitly depends on the velocity of the fluid with respect to the wall. Thus, Eq. (17) gives the balance between the forces that rule the stationary wall motion.

While the integration of Eqs. (1) across the wall was straightforward, in this case we shall need some approximations to avoid the dependence on the wall shape [28]. To evaluate the last integral in Eq. (7), we notice that $\phi^{\prime}(z)^{2}$ behaves approximately like a delta function which picks the value of $\gamma v$ at the center of the wall width, say $\gamma_{0} v_{0}$. Thus, the integral gives $\gamma_{0} v_{0} \sigma$, where $\sigma \equiv \int \phi^{\prime 2} d z$ is the surface tension. We shall approximate the value of $\gamma_{0} v_{0}$ by the average $\langle\gamma v\rangle \equiv \frac{1}{2}\left(\gamma_{+} v_{+}+\gamma_{-} v_{-}\right)$. Thus, the last term in (17) can be written in the form $-\eta\langle\gamma v\rangle$, with

$$
\eta=\tilde{\eta} T_{c} \sigma
$$

and the force balance reads

$$
\eta\langle\gamma v\rangle=-F_{\mathrm{dr}} .
$$

For the first integral in Eq. (7), we can use a linear approximation for the $z$ dependence of the entropy density $s=-\partial \mathcal{F} / \partial T$ inside the wall. We obtain $\frac{1}{2}\left(s_{+}+s_{-}\right)\left(T_{+}-T_{-}\right)$. This gives the following approximation for the driving force,

$$
F_{\mathrm{dr}}=p_{-}\left(T_{-}\right)-p_{+}\left(T_{+}\right)+\langle s\rangle\left(T_{+}-T_{-}\right)
$$

In many cases, the free energy has even powers of the temperature, whereas the last term in Eq. (11) introduces odd powers. A more convenient approximation for such cases can be obtained by noticing that $(\partial \mathcal{F} / \partial T) d T=\left(\partial \mathcal{F} / \partial T^{2}\right) d T^{2}$. Then, instead of using a linear approximation for $\partial \mathcal{F} / \partial T$, we may use a linear approximation for $\partial \mathcal{F} / \partial T^{2}$. We obtain

$$
F_{\mathrm{dr}}=p_{-}\left(T_{-}\right)-p_{+}\left(T_{+}\right)+\left\langle\frac{d p}{d T^{2}}\right\rangle\left(T_{+}^{2}-T_{-}^{2}\right) .
$$

The approximations (11) and (12) are quantitatively very similar, though analytically different. The former involves the physical quantity $s$ and is useful for physical discussions. As we shall see, the latter gives cleaner analytical results. 


\section{STABILITY ANALYSIS}

\section{A. Previous results and relevant scales}

The stability of deflagrations in a cosmological phase transition was first studied by Link [13] in the non-relativistic limit. The results are similar to those of a classical gas [12] (with the enthalpy taking the role of the mass density). According to this analysis, small wavelength perturbations of the phase transition front are stabilized by the surface tension $\sigma$, whereas large wavelength perturbations grow exponentially. The perturbations are of the form

$$
e^{i \mathbf{k} \cdot \mathbf{x}^{\perp}+q z+\Omega t}
$$

and the initial growth time is given by $\Omega^{-1}$. The essential features of the instability are easily seen if we consider the limit $T_{ \pm} \simeq T_{c}$ in Link's result, so that the latent heat is given by $L \simeq w_{+}-w_{-}$. For small $L / w_{+}$we have

$$
\Omega \simeq \frac{L}{w_{+}} \frac{v_{w}}{2}\left(1-\frac{k}{k_{c}}\right) k
$$

with

$$
k_{c}=L v_{w}^{2} / \sigma
$$

Thus, the critical wavelength above which perturbations are unstable is given by $\lambda_{c}=1 / k_{c}$. Notice that, for non-relativistic velocities, Eqs. (55-6) give $p_{+}-p_{-}=L v_{+} v_{-}$which, for small $L / w_{+}$becomes $p_{+}-p_{-}=L v_{w}^{2}$. Therefore, the critical wavelength can be written as

$$
\lambda_{c}=\frac{\sigma}{p_{+}\left(T_{+}\right)-p_{-}\left(T_{-}\right)} \equiv d_{c}
$$

Physically, $d_{c}$ is the length scale over which the surface tension just balances the difference of the pressures on each side of the interface [12, 13].

This result was improved by Huet et al. [14]. In the first place, relativistic velocities were considered in order to study fast deflagrations $\left(v_{w} \rightarrow c_{s}\right)$. Most important, the dependence of the wall velocity on temperature was taken into account. A very simple form for the velocity was considered.

$$
\gamma_{w} v_{w}\left(T_{+}\right)=\frac{p_{-}\left(T_{+}\right)-p_{+}\left(T_{+}\right)}{\eta} \approx \frac{L\left(1-T_{+} / T_{c}\right)}{\eta} .
$$

where $\eta$ is a friction parameter, which can be assumed to be a constant. The last approximation in Eq. (17) is valid in the non-relativistic limit and for $T_{+}$close to $T_{c}$. The inclusion of Eq. (17) into consideration takes into account that temperature fluctuations induce a change in the velocity of the interface. Instead of this, the treatment of Landau [12] just equated the normal velocity fluctuations $\delta v_{ \pm}$of the fluid to the velocity of the surface perturbation $\partial_{0} \zeta$, while Link [13] considered the energy flux $F=w v \gamma^{2}$ to be proportional to the net blackbody energy flux, $F \propto g_{-}\left(\pi^{2} / 30\right)\left(T_{+}^{4}-T_{-}^{4}\right)$, where $g_{-}$is the effective number of degrees of freedom in the - phase. Notice that these conditions lack important information, namely, that the interface is a phase transition front (and not, e.g., a burning front). This information is not present in either of the equations considered in [12, 13]. In contrast, in Eq. (17), the velocity of the interface depends on the pressure difference and vanishes at $T_{+}=T_{c}$, which is the essential feature of a phase transition. 
From Eq. (17), an important parameter arises in the perturbation equations, namely,

$$
\beta=T_{c}\left(-\frac{d v_{w}}{d T_{+}}\right) \gamma_{w}^{2} v_{w}
$$

The dependence of the wall velocity on temperature tends to stabilize the perturbations. In the non-relativistic limit and for $\Omega \ll k$, the result of Ref. [14] takes the form

$$
\Omega \simeq \frac{\bar{L} v_{w}}{2} \frac{\lambda-\lambda_{c}}{\lambda^{2}} \frac{(1-\beta)}{1+\beta \bar{L}+d_{b} k}
$$

with

$$
\lambda_{c}=d_{c}\left(1+\frac{2 d_{b} / d_{c}}{\bar{L}(1-\beta)}\right),
$$

where $\bar{L}=L / w_{+}$, and a new length scale

$$
d_{b}=\frac{\sigma}{p_{-}\left(T_{+}\right)-p_{+}\left(T_{+}\right)}
$$

has appeared due to the introduction of Eq. (17). Its physical significance is similar to that of $d_{c}$ [cf. Eq. (16)]. It gives the length scale over which the surface tension just balances the pressure difference in equilibrium at $T=T_{+}$. Thus, it gives the size scale of a critical bubble before it begins to grow. As remarked in Ref. [14], the quantity $d_{b}$ gives also a time scale characteristic of the dynamics of growth, corresponding to the acceleration period before the bubble wall reaches a terminal velocity. Within the present approximations, the two scales $d_{b}$ and $d_{c}$ are related by

$$
\frac{d_{b}}{d_{c}}=\frac{v_{w}^{2}}{1-T_{+} / T_{c}}
$$

and Eq. (18) gives $\beta=v_{w} L / \eta$. We can eliminate the friction parameter and write [14]

$$
\beta=v_{w}^{2} / v_{c}^{2}
$$

where

$$
v_{c}^{2} \equiv\left(T_{c}-T_{+}\right) / T_{c}
$$

The length scales are thus related by $d_{b} / d_{c}=\beta$.

A few comments on these scales are worth. Notice that, in the equilibrium situation with $T_{-}=T_{+}$, we have $p_{-}\left(T_{+}\right)>p_{+}\left(T_{+}\right)$, whereas, for a real deflagration with $T_{+}>T_{-}$(see Fig. 2), the pressure balance is inverted, $p_{-}\left(T_{-}\right)<p_{+}\left(T_{+}\right)$(this is why it is necessary to invert the order of $p_{-}$and $p_{+}$between the definitions of $d_{c}$ and $\left.d_{b}\right)$. It is evident that the pressure difference $p_{-}\left(T_{-}\right)-p_{+}\left(T_{+}\right)$, being negative, cannot be used as the driving force, and the equilibrium value $p_{-}\left(T_{+}\right)-p_{+}\left(T_{+}\right)$in Eq. (17) is a better approximation (although it does not take hydrodynamics into account). A still better approximation to the driving force is given by Eq. (11).

In practice, the relevant difference between the results of Refs. [13] and [14] is the appearance of the quantity $(1-\beta)$ in Eqs. (19,20) with respect to Eqs. (14-16). For $\beta \ll 1$, the results are essentially the same. However, for $\beta \approx 1$ the situation changes drastically. For $\beta<1$, perturbations on wavelengths $\lambda<\lambda_{c}$ are stable. Thus, as $\beta$ approaches 1 , only very large wavelengths will be unstable, and with very large growth time $\Omega^{-1}$. For $\beta>1$, 
the behavior is inverted. Perturbations with $\lambda>\lambda_{c}$ are now stable. Besides, the second term in Eq. (201) dominates, and we have $\lambda_{c}<0$. This means that perturbations at all scales are stable for $\beta>1$. Hence, $v_{c}$ is a critical velocity, above which the deflagration becomes stable.

Notice that the second term in Eq. (20) is not a small correction, even for $\beta \ll 1$. In fact, due to the smallness of $\bar{L}$, this term will generally dominate. As a consequence, the critical wavelength predicted by Huet et al. is generally quite larger than Link's result. A numerical study of the stability of planar walls was performed in Ref. [15] for the QCD phase transition, for a value $\bar{L} \approx 0.089$. For the case of deflagrations, two cases were considered, corresponding to $v_{w}=0.196$ (case A) and $v_{w}=0.048$ (case B). For each of these two runs of the numerical simulation, the planar wall was initialized with sinusoidal perturbations of wavelengths up to $\lambda=5 \lambda_{c}^{\text {Link }}$, where $\lambda_{c}^{\text {Link }}$ is the critical wavelength obtained by Link and given approximately by Eq. (16). According to Link's results, these perturbations should be unstable. However, the perturbations decayed in the simulation, in agreement with the results of Huet et al. Indeed, the stability parameter is $\beta=0.516$ for case $\mathrm{A}$ and $\beta=0.722$ for case B [45]. Hence, according to Eq. (20), we have $\lambda_{c} \approx 25 \lambda_{c}^{\text {Link }}$ and $\lambda_{c} \approx 60 \lambda_{c}^{\text {Link }}$, respectively. Perturbations of wavelengths $\lambda$ higher than these values of $\lambda_{c}$ should be unstable according to the results of Huet et al. Unfortunately, such perturbations were not considered in the simulations of Ref. [15].

Although the treatment of Ref. [14] improved significantly upon previous stability analysis, some of the approximations used in this approach will not hold in the general situation. The most important are the following.

In the first place, we remark that the simple expression (17) for $v_{w}$ implicitly assumes the relations $T_{-}=T_{+}$and $v_{+}=v_{-}=v_{w}$ [cf. Eqs. (10), (11)], while for a deflagration we have $T_{-}<T_{+}$and $v_{+}<v_{-}=v_{w}$. Moreover, since the expression (17) depends only on $T_{+}$ and $v_{-}$, it does not allow to perform independent perturbations of variables on each side of the wall (such as $\delta T_{-}, \delta v_{+}$). These limitations of the surface equation constrain the validity of the treatment of Huet et al. This contrasts with their treatment of the fluid equations, where independent perturbations were considered for - and + variables. One expects that the approximation $\delta T_{-}=\delta T_{+}$will be valid if $T_{-} \simeq T_{+}$. However, the latter is not the most general case.

In the second place, the reheating in front of the wall was not taken into account. The value of $T_{+}$was estimated from results on the amount of supercooling ${ }^{3}$ for the electroweak phase transition [9] (i.e., the approximation $T_{+} \simeq T_{N}$ was used). Notice that, for a deflagration, the fluid is reheated in front of the wall (see Fig. 2). As a consequence, the temperature $T_{+}$in front of the phase transition front does not coincide with the nucleation temperature $T_{N}$. Depending on the wall velocity and the amount of latent heat released, the local reheating can be important.

Our derivation of the perturbation equations will be similar to that of Ref. [14]. The main difference will be, essentially, that instead of considering Eq. (17), we shall consider Eq. (10), which depends explicitly on the two velocities $v_{ \pm}$and on the two temperatures $T_{ \pm}$ (through the driving force). According to the approximation (11), we have $F_{\mathrm{dr}}=p_{-}\left(T_{-}\right)-$ $p_{+}\left(T_{+}\right)+\langle s\rangle\left(T_{+}-T_{-}\right)$. In the case of small supercooling (i.e., $T_{c}-T_{ \pm} \ll T_{c}$ ) and small wall velocity, the pressure difference is $\mathcal{O}\left(L v_{w}^{2}\right)$ and can be neglected in comparison with the

\footnotetext{
${ }^{3}$ The temperature at which bubbles nucleate and expand can be estimated using the bubble nucleation rate, which is calculated using the thermal instanton technique [30].
} 
term $\langle s\rangle\left(T_{+}-T_{-}\right)$. Besides, we can use the approximation [28]

$$
T_{+}-T_{-}=\frac{\Delta s\left(T_{c}\right)}{s_{-}\left(T_{c}\right)}\left(T_{c}-T_{+}\right)
$$

to obtain

$$
F_{\mathrm{dr}}=\left(\langle w\rangle / w_{-}\right) L\left(1-T_{+} / T_{c}\right),
$$

which, taking into account that $\langle v\rangle=-v_{w}\langle w\rangle / w_{+}$, gives

$$
v_{w}=\frac{w_{+}}{w_{-}} \frac{L\left(1-T_{+} / T_{c}\right)}{\eta} .
$$

This is similar to Eq. (17), except for the factor $w_{+} / w_{-}$, which is $\approx 1$ for small latent heat. Thus, in the limit of small supercooling, small $v_{w}$, and small $L / w_{+}$, we obtain the approximation used in Ref. [14] for the wall velocity. In our treatment, the parameter $d_{b}$ will be replaced by

$$
d=\frac{\sigma}{F_{\mathrm{dr}}}
$$

For small supercooling and small latent heat, we have $d_{b} \approx d$. According to Eq. (22), the parameter $d_{c}$ is determined by $d_{b}$ and $v_{c}$. Similarly, our results will depend on $d$ and the critical velocity.

\section{B. Linearized equations}

We shall consider small perturbations of the fluid and the interface. The planar symmetry allows us to consider a single transverse direction $x^{\perp}$ instead of two directions $x, y$. The perturbation variables we shall use are the pressure fluctuation $\delta p\left(x^{\perp}, z, t\right)$, the variation of the velocity along the wall motion $\delta v\left(x^{\perp}, z, t\right)$, the transverse velocity $v^{\perp}\left(x^{\perp}, z, t\right)$, and the variation of the wall position $\zeta\left(x^{\perp}, t\right)$. For the sake of clarity, in this subsection we shall denote the stationary solutions with a bar. Thus, we have

$$
\begin{aligned}
p & =\bar{p}+\delta p, \\
u^{\mu} & =\bar{u}^{\mu}+\delta u^{\mu}=(\bar{\gamma}, 0,0, \bar{\gamma} \bar{v})+\left(\bar{\gamma}^{3} \bar{v} \delta v, \bar{\gamma} v^{\perp}, \bar{\gamma} \delta v\right), \\
z_{w} & =\bar{z}_{w}+\zeta .
\end{aligned}
$$

To derive the equations for these four variables, we shall consider again Eqs. (11-2) for a field configuration corresponding to the perturbed wall.

\section{Fluid equations}

Away from the (perturbed) interface, the field is a constant as before, and Eq. (11) gives the local conservation of energy and momentum $\partial_{\mu} T^{\mu \nu}=0$, where $T^{\mu \nu}$ is given by Eq. (3). To obtain the fluid equations it is convenient to take the projections of the 4divergence of the stress tensor along the directions of the fluid 4-velocity and orthogonal to it, $u_{\mu} T^{\mu \nu}{ }_{, \nu}=0,\left(g_{\alpha \mu}-u_{\alpha} u_{\mu}\right) T^{\mu \nu}{ }_{, \nu}=0$. Taking into account the relation between enthalpy and pressure variations, Eq. (4), we obtain

$$
\begin{array}{r}
c_{s}^{2} w u_{, \nu}^{\nu}+u^{\nu} p_{, \nu}=0, \\
w u_{\alpha, \nu} u^{\nu}-p_{, \alpha}+u_{\alpha} u^{\nu} p_{, \nu}=0 .
\end{array}
$$


We are interested in the stability of the deflagration solution depicted in Fig. 2, for which the fluid profile is constant on both sides of the wall. Therefore, we must consider perturbations from a constant solution (we shall not consider here perturbations of the shock front). To linear order in the perturbations we obtain, for the direction along the fluid velocity,

$$
c_{s}^{2} \bar{w}\left(\bar{\gamma}^{2} \bar{v} \delta v,_{0}+\bar{\gamma}^{2} \delta v_{, z}+v_{, \perp}^{\perp}\right)+\delta p_{, 0}+\bar{v} \delta p_{, z}=0
$$

and, for the orthogonal directions,

$$
\begin{aligned}
\bar{w} \bar{\gamma}^{2}\left(\delta v_{, 0}+\bar{v} \delta v_{, z}\right)+\bar{v} \delta p_{, 0}+\delta p_{, z} & =0 \\
\bar{w} \bar{\gamma}^{2}\left(v_{, 0}^{\perp}+\bar{v} v_{, z}^{\perp}\right)+\delta p_{, \perp} & =0 .
\end{aligned}
$$

We have not specified the reference frame yet. We shall consider the frame which moves with the unperturbed wall. Therefore, $\bar{v}$ corresponds to the incoming and outgoing flow velocities.

\section{Matching conditions at the interface}

To obtain matching conditions for the perturbations, we need to derive the matching conditions for the perturbed wall, and then subtract those for the stationary wall. We shall consider that the unperturbed wall is at $\bar{z}_{w}=0$ and the perturbed wall at $z_{w}=\zeta\left(x^{\perp}, t\right)$. Let us consider again Eqs. (1), $\partial_{\mu}\left(w u^{\mu} u^{\nu}-g^{\mu \nu} p\right)=-\square \phi \partial^{\nu} \phi$.

Since there is a discontinuity at the bubble wall, if we integrate these equations in a small interval along the normal direction across the surface, only the normal derivatives will give a finite difference. Thus we can neglect all other derivatives. We obtain

$$
\partial_{n}\left(w \gamma^{2} v^{n}\right)=0, \quad \partial_{n}\left(w \gamma^{2} v^{n} v^{\perp}\right)=0, \quad \partial_{n}\left(w \gamma^{2} v^{n 2}+p\right)=\square \phi \partial_{n} \phi .
$$

For instance, for the unperturbed wall we have $n=z, \square \phi=-\partial_{z}^{2} \phi$, and we re-obtain Eqs. (5+6),

$$
\begin{aligned}
\Delta\left(\bar{w} \bar{v} \bar{\gamma}^{2}\right) & =0 \\
\Delta\left(\bar{w} \bar{v}^{2} \bar{\gamma}^{2}+\bar{p}\right) & =0
\end{aligned}
$$

together with the continuity of the transverse velocity, $\Delta \bar{v}^{\perp}=0$. The velocity $\bar{v}^{\perp}$, though, is set to zero by symmetry. If we use Eqs. (37) for the perturbed wall, we then have to express $\partial_{n}$ in terms of $\partial_{z}, \partial_{\perp}$ and $\partial_{0}$, in order to compare with the stationary equations.

Alternatively, we may stay in the coordinate system of the unperturbed wall and just consider the fluid equations,

$$
\begin{aligned}
\partial_{0}\left(w \gamma^{2}-p\right)+\partial_{z}\left(w \gamma^{2} v\right)+\partial_{\perp}\left(w \gamma^{2} v^{\perp}\right) & =-\square \phi \partial_{0} \phi, \\
\partial_{0}\left(w \gamma^{2} v^{\perp}\right)+\partial_{z}\left(w \gamma^{2} v v^{\perp}\right)+\partial_{\perp}\left(w \gamma^{2} v^{\perp}+p\right) & =\square \phi \partial_{\perp} \phi, \\
\partial_{0}\left(w \gamma^{2} v\right)+\partial_{z}\left(w \gamma^{2} v^{2}+p\right)+\partial_{\perp}\left(w \gamma^{2} v v^{\perp}\right) & =\square \phi \partial_{z} \phi,
\end{aligned}
$$

taking into account the discontinuity of the variables at the wall. We shall use this approach. We are going to integrate across the wall along the $z$ axis. Since we shall integrate in a vanishingly small interval, we may neglect any dependence on $x^{\perp}$ and $t$, other than the position of the discontinuity, i.e., we may assume (for this integration) step functions depending only 
on $z-\zeta\left(x^{\perp}, t\right)$. Hence, we have, e.g., $\partial_{0} w=-\partial_{0} \zeta \partial_{z} w, \partial_{\perp} w=-\partial_{\perp} \zeta \partial_{z} w$, etc. This leaves only $z$ derivatives in the lhs of Eqs. (40,42).

Keeping up to linear terms in the perturbations $\zeta$ and $v^{\perp}$, only terms proportional to $\partial_{z}^{2} \phi \partial_{z} \phi$ (which vanish after integration) remain in the rhs, except in Eq. (42), where there is also a term proportional to $\left(\partial_{z} \phi\right)^{2}$. Performing the $z$ integration, the latter gives the surface tension $\sigma=\int\left(\partial_{z} \phi\right)^{2} d z$. We thus obtain

$$
\begin{aligned}
-\partial_{0} \zeta \Delta\left(w \gamma^{2}-p\right)+\Delta\left(w \gamma^{2} v\right) & =0 \\
\Delta\left(w \gamma^{2} v v^{\perp}\right)-\partial_{\perp} \zeta \Delta p & =0 \\
\Delta\left(w \gamma^{2} v^{2}+p\right) & =-\sigma\left(\partial_{0}^{2}-\partial_{\perp}^{2}\right) \zeta
\end{aligned}
$$

where $\Delta$ applied to any function $f$ means $\Delta f=f_{+}-f_{-}$. In the last equation we have used the fact that, according to Eq. (43),$\Delta\left(w \gamma^{2} v\right)=\mathcal{O}(\zeta)$. Now we replace $w=\bar{w}+\delta w$, $v=\bar{v}+\delta v$, etc., taking into account the unperturbed equations (38) 39) and the relation $\delta w=\left(1+c_{s}^{-2}\right) \delta p$. To first order in all the perturbations, we have

$$
\begin{aligned}
\Delta\left[\bar{w} \bar{\gamma}^{2}\left(1+\bar{v}^{2}\right)\left(-\partial_{0} \zeta+\bar{\gamma}^{2} \delta v\right)+\left(1+c_{s}^{-2}\right) \bar{\gamma}^{2} \bar{v} \delta p\right] & =0, \\
\Delta\left(v^{\perp}+\bar{v} \partial_{\perp} \zeta\right) & =0, \\
\sigma\left(\partial_{0}^{2}-\partial_{\perp}^{2}\right) \zeta+\Delta\left[2 \bar{w} \bar{\gamma}^{4} \bar{v} \delta v+\left(1+\left(1+c_{s}^{-2}\right) \bar{\gamma}^{2} \bar{v}^{2}\right) \delta p\right] & =0 .
\end{aligned}
$$

\section{Equation for the interface}

Let us now consider the field equation (2). The field varies in a region (the wall width) around the wall position $z_{w}=\zeta\left(x^{\perp}, t\right)$. Hence, we may assume a field profile of the form ${ }^{4}$ $\phi\left(z, x^{\perp}, t\right)=\phi\left[z-\zeta\left(x^{\perp}, t\right)\right]$. To first order in $\zeta$ and $v^{\perp}$, we have $\partial_{\mu} \partial^{\mu} \phi=\phi^{\prime}\left(\partial_{\perp}^{2}-\partial_{0}^{2}\right) \zeta-\phi^{\prime \prime}$ and $u^{\mu} \partial_{\mu} \phi=\gamma\left(-\partial_{0} \zeta+v\right) \phi^{\prime}$. Multiplying Eq. (2) by $\phi^{\prime}(z-\zeta)$ and integrating in $z$ (as we did in Sec. II), we obtain

$$
\sigma\left(\partial_{0}^{2}-\partial_{\perp}^{2}\right) \zeta=p_{-}-p_{+}+\int s d T+\tilde{\eta} T_{c} \int \phi^{2} \gamma\left(v-\partial_{0} \zeta\right) d z .
$$

This equation is similar to Eq. (7), except for the three terms depending on $\zeta$. The first one, $\sigma \partial_{0}^{2} \zeta$, takes into account the acceleration of a surface element of the wall which, according to Eq. (49), is determined by the sum of all the forces acting on it. The second one, $-\sigma \partial_{\perp}^{2} \zeta$, gives the restoring force due to the curvature of the surface. Finally, the term $-\partial_{0} \zeta$ takes into account the fact that the friction force depends on the relative velocity $v_{r}$ between the fluid and the wall. We have $\gamma\left(v-\partial_{0} \zeta\right)=\gamma_{r} v_{r}$.

Approximating the integrals in (49) as we did in Sec. III, we obtain

$$
\sigma\left(\partial_{0}^{2}-\partial_{\perp}^{2}\right) \zeta-F_{\mathrm{dr}}-\eta\left\langle\gamma\left(v-\partial_{0} \zeta\right)\right\rangle=0 .
$$

The various thermodynamical quantities (entropy, pressure, temperature), are related through the equation of state. Hence, we may consider the driving force as a function of $T_{-}$and $T_{+}$. In the stationary case, Eq. (50) gives Eq. (10),

$$
\langle\bar{\gamma} \bar{v}\rangle=-F_{\mathrm{dr}}\left(\bar{T}_{+}, \bar{T}_{-}\right) / \eta
$$

\footnotetext{
${ }^{4}$ Notice that here we are considering a reference frame for which $\bar{z}_{w}=\dot{\bar{z}}_{w}=0$, and $\zeta$ is a small perturbation, so we have $\gamma_{w}=1+\mathcal{O}\left(\zeta^{2}\right)$.
} 
where $\bar{T}_{-}$and $\bar{T}_{+}$are related through Eqs. (38)[39). For the perturbations we obtain

$$
\sigma\left(\partial_{0}^{2}-\partial_{\perp}^{2}\right) \zeta=\eta\left\langle\delta(\gamma v)-\bar{\gamma} \partial_{0} \zeta\right\rangle+\frac{\partial F_{\mathrm{dr}}}{\partial T_{+}} \delta T_{+}+\frac{\partial F_{\mathrm{dr}}}{\partial T_{-}} \delta T_{-}
$$

The derivatives $\partial F_{\mathrm{dr}} / \partial T_{ \pm}$can be calculated by using either the approximation (11) or the approximation (12). In terms of our perturbation variables $\delta p_{ \pm}$, we have

$$
\frac{\sigma}{\eta}\left(\partial_{0}^{2}-\partial_{\perp}^{2}\right) \zeta=\frac{1}{2}\left[\delta\left(\gamma_{+} v_{+}\right)+b_{+} \frac{\delta p_{+}}{w_{+}}-\bar{\gamma}_{+} \partial_{0} \zeta\right]+\frac{1}{2}\left[\delta\left(\gamma_{-} v_{-}\right)+b_{-} \frac{\delta p_{-}}{w_{-}}-\bar{\gamma}_{-} \partial_{0} \zeta\right]
$$

where

$$
\frac{b_{ \pm}}{2} \equiv T_{ \pm} \frac{1}{\eta} \frac{\partial F_{\mathrm{dr}}}{\partial T_{ \pm}}
$$

The parameters $\sigma$ and $\eta$ can be written in terms of the fluid velocity and the scale $d$ using Eqs. (51) and (28). Thus, we can write Eq. (53) in a more concise form,

$$
\left\langle\bar{\gamma} \bar{v} d\left(\partial_{0}^{2}-\partial_{\perp}^{2}\right) \zeta+\bar{\gamma}^{3} \delta v+b \delta p / w-\bar{\gamma} \partial_{0} \zeta\right\rangle=0
$$

with

$$
\frac{b_{ \pm}}{2}=\langle-\bar{\gamma} \bar{v}\rangle \frac{T_{ \pm}}{F_{\mathrm{dr}}} \frac{\partial F_{\mathrm{dr}}}{\partial T_{ \pm}}=2\langle-\bar{\gamma} \bar{v}\rangle \frac{T_{ \pm}^{2}}{F_{\mathrm{dr}}} \frac{\partial F_{\mathrm{dr}}}{\partial T_{ \pm}^{2}} .
$$

The last equality is useful if $F_{\mathrm{dr}}$ is quadratic in temperature.

To understand the meaning of these equations, it is useful to consider some simplifications used in previous works. In Ref. [14] hydrodynamics was neglected in the equation for the wall. This means that, in the first place, it was assumed that $\bar{T}_{-}=\bar{T}_{+}$and $\bar{v}_{+}=\bar{v}_{-}=-v_{w}$. In this case we have $F_{\mathrm{dr}}=p_{-}\left(\bar{T}_{+}\right)-p_{+}\left(\bar{T}_{+}\right)$and the scale $d$ becomes equal to $d_{b}$ defined in (21). In the second place, only linearly-dependent perturbations were considered for the interface equation, $\delta T_{-}=\delta T_{+}, \delta v_{-}=\delta v_{+}$, such that even the perturbed force is of the form $F_{\mathrm{dr}}\left(T_{+}\right)=p_{-}\left(T_{+}\right)-p_{+}\left(T_{+}\right)$. This is the most sensitive simplification used in Ref. [14], since perturbations in each phase may be quite different ${ }^{5}$. With these approximations, the two terms in the rhs of (53) are almost identical. The sum of the two coefficients $b_{ \pm} / 2$ contains a total derivative $d F_{\mathrm{dr}} / d T_{+}$and gives

$$
\langle b\rangle=T_{+} \frac{d\left(\gamma_{w} v_{w}\right)}{d T_{+}} .
$$

We thus obtain

$$
v_{w} d_{b}\left(\partial_{0}^{2}-\partial_{\perp}^{2}\right) \zeta=(1-\beta) \gamma_{+}^{2} \delta v_{+}-\partial_{0} \zeta
$$

where

$$
\beta=-T_{+} \frac{\partial v_{w}}{\partial T_{+}} \frac{1}{w_{+}} \frac{\delta p_{+}}{\delta v_{+}}
$$

In Eq. (59), the variation $\delta p / \delta v$ depends on the solution of the fluid equations (34-36) on the + side of the wall.

${ }^{5}$ This is more apparent in the case of detonations, for which perturbations can grow only in the - phase, and leads the authors of Ref. [14] to a wrong conclusion about the stability of detonations [16, 31]. 
Equation (58) is essentially ${ }^{6}$ the same as Eq (52) of Ref. [14]. As we have seen at the beginning of this section, the coefficient $\beta$ plays a relevant role in the hydrodynamic stability of the deflagration. In the realistic case, we see that $\beta$ will split into two parts, $\beta_{ \pm}$, corresponding to perturbations on each side of the wall. We remark that, in the case of small supercooling, Eq. (26) (which depends only on $T_{+}$) is a good approximation for the stationary force $F_{\mathrm{dr}}\left(\bar{T}_{+}, \bar{T}_{-}\right)$, but not for the general form $F_{\mathrm{dr}}\left(T_{+}, T_{-}\right)$, and should not be used to obtain $b_{ \pm}$.

\section{Fourier modes of the perturbations}

The fluid equations away from the wall, Eqs. (34 36), can be expressed in matrix form,

$$
\left[C_{0} \partial_{0}+C_{z} \partial_{z}+C_{\perp} \partial_{\perp}\right] \vec{U}=0
$$

where (from now on we remove the bars on unperturbed variables)

$$
C_{0} \equiv\left[\begin{array}{ccc}
1 & c_{s}^{2} w \gamma^{2} v & 0 \\
v & w \gamma^{2} & 0 \\
0 & 0 & w \gamma^{2}
\end{array}\right], C_{z} \equiv\left[\begin{array}{ccc}
v & c_{s}^{2} w \gamma^{2} & 0 \\
1 & w \gamma^{2} v & 0 \\
0 & 0 & w \gamma^{2} v
\end{array}\right], C_{\perp} \equiv\left[\begin{array}{ccc}
0 & 0 & c_{s}^{2} w \\
0 & 0 & 0 \\
1 & 0 & 0
\end{array}\right]
$$

and $\vec{U}$ is the perturbation vector

$$
\vec{U} \equiv\left[\begin{array}{c}
\delta p \\
\delta v \\
v^{\perp}
\end{array}\right]
$$

To solve Eq. (60) we may use the separation of variables method which, in this simple case, amounts to searching for solutions of the form

$$
\vec{U}\left(t, z, x^{\perp}\right)=\vec{L} e^{\Omega t+q z+i k x^{\perp}},
$$

where $\Omega, q$ and $i k$ are the eigenvalues of the operators $\partial_{0}, \partial_{z}$ and $\partial_{\perp}$, respectively. While $k$ is a real wavenumber, corresponding to Fourier modes along the wall, $\Omega$ and $q$ are in general complex numbers. The stationary solution will be unstable whenever $\operatorname{Re}(\Omega)>0$.

Inserting the modes (63) into Eqs. (60) we obtain the system of homogeneous equations

$$
\left(C_{0} \Omega+C_{z} q+C_{\perp} i k\right) \vec{L} \equiv C \vec{L}=0
$$

The determinant of the matrix $C$ must vanish so that the trivial solution is not the only one. This gives the dispersion relations

$$
q v+\Omega=0
$$

or

$$
(q v+\Omega)^{2}-c_{s}^{2}(\Omega v+q)^{2}+c_{s}^{2} \gamma^{-2} k^{2}=0
$$

\footnotetext{
${ }^{6}$ There is a discrepancy, namely, the relative factor of $\gamma_{+}^{2}$ between the terms $\delta v_{+}$and $\partial_{0} \zeta$. The origin of this is that, in their derivation, the authors of [14] considered, for $v_{+} \approx-v_{w}$, the relation $\delta\left(v_{+}+v_{w}\right)=\partial_{0} \zeta$. However, the correct relativistic velocity sum gives $\delta\left(\gamma_{+}^{2}\left(v_{+}+v_{w}\right)\right)=\partial_{0} \zeta$.
} 
and we have three solutions,

$$
\begin{aligned}
q_{1} & =-\Omega / v \\
q_{2,3} & =\frac{\left(1-c_{s}^{2}\right) v \Omega \pm c_{s}\left(1-v^{2}\right) \sqrt{\Omega^{2}+\left(c_{s}^{2}-v^{2}\right) \gamma^{2} k^{2}}}{c_{s}^{2}-v^{2}} .
\end{aligned}
$$

The corresponding eigenvectors are

$$
\vec{L}_{1}=\left[\begin{array}{c}
0 \\
1 \\
\frac{i q_{1}}{k}
\end{array}\right], \vec{L}_{2,3}=\left[\begin{array}{c}
-w \gamma^{2}\left(\frac{\Omega+q_{2,3} v}{\Omega v+q_{2,3}}\right) \\
1 \\
\frac{i k}{\Omega v+q_{2,3}}
\end{array}\right]
$$

The eigenvector $\vec{L}_{1}$ corresponds to a special solution which describes isobaric perturbations $\left(\delta p_{1}=0\right)$ moving with the fluid (i.e., with $z, t$ dependence of the form $\left.z-v t\right)$.

The general solution is a superposition of these modes. In particular, for given $k$ and $\Omega$, we must consider a perturbation vector of the form

$$
\vec{U}\left(t, z, x^{\perp}\right)=\vec{A}(z) e^{\left(\Omega t+i k x^{\perp}\right)},
$$

with

$$
\vec{A}(z)=\sum_{j=1}^{3} A_{j} \vec{L}_{j} e^{q_{j} z} .
$$

The function $\vec{A}(z)$ must satisfy the boundary conditions at $z= \pm \infty$ and the junction conditions at the wall. Accordingly, the perturbation from the planar shape of the surface will be of the form

$$
\zeta\left(t, x^{\perp}\right)=D e^{\left(\Omega t+i k x^{\perp}\right)} .
$$

For $\operatorname{Re}(\Omega)>0$ the perturbation grows exponentially with time, whereas for $\operatorname{Re}(\Omega)<0$ the perturbation decays exponentially. On the other hand, the condition that the source is the wall itself, and not something outside it, implies that the perturbations must decay away from the wall [12]. Therefore, instability of the front also requires $\operatorname{Re}(q)<0$ for $z>0$ (+ phase) and $\operatorname{Re}(q)>0$ for $z<0$ ( - phase).

Since $v$ is negative, the special solution gives $\operatorname{Re}\left(q_{1}\right)>0$ for $\operatorname{Re}(\Omega)>0$. Hence, unstable perturbations will be associated to the presence of this mode in the - phase (behind the wall). Conversely, for stable perturbations this mode will be in the + phase.

Regarding the other two solutions, we can write Eq. (68) in the form $\left(\Omega-a_{+} q\right)\left(\Omega-a_{-} q\right)=$ $-K^{2}$, with

$$
a_{ \pm}= \pm \frac{c_{s} \mp v}{1 \mp c_{s} v}
$$

and $K^{2}=c_{s}^{2} \gamma^{-2} k^{2} /\left(1-c_{s}^{2} v^{2}\right)$, which shows that, for real $q$ and $\Omega$, we have a hyperbola with asymptotes $q=\Omega / a_{ \pm}$. For complex $q$ and $\Omega$, it can be shown that the points $\operatorname{Re}(q), \operatorname{Re}(\Omega)$ lie in the same region between these asymptotes. We show some examples in Fig. 4. We have three different cases, depending on the value of $v$. For $v$ supersonic (left panel), $\operatorname{Re}\left(q_{2}\right)$ and $\operatorname{Re}\left(q_{3}\right)$ have the same sign for a given $\Omega$. For $v$ subsonic (right panel), $\operatorname{Re}\left(q_{2}\right)$ and $\operatorname{Re}\left(q_{3}\right)$ have opposite sign. In the case $v=-c_{s}$ (central panel) there is only one solution (besides the special one), namely

$$
q_{2}=\frac{c_{s} k^{2}}{2 \Omega}+\frac{\left(1+c_{s}^{2}\right)}{2 c_{s}} \Omega
$$


In front of the wall we require $\operatorname{Re}(q)<0$, and the possible modes are those in the lower quadrants of Fig. 4. Conversely, the possible modes behind the wall are those in the upper quadrants.
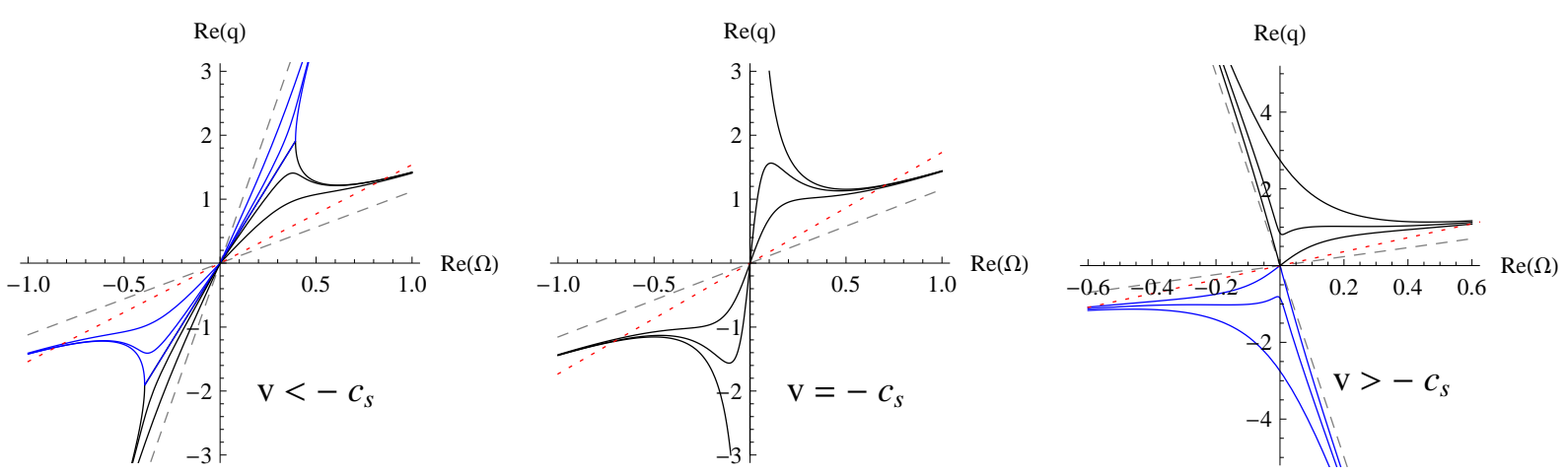

FIG. 4: The real part of the dispersion relations $q_{2}(\Omega)$ (in black) and $q_{3}(\Omega)$ (in blue) for different values of the imaginary part $\operatorname{Im}(\Omega)$. Gray dashed lines indicate the asymptotes of these solutions. We also show the special solution $q_{1}(\Omega)$ in a dotted red line.

Deflagrations are characterized by $\left|v_{+}\right|<c_{s+}$, i.e., the incoming flow is always subsonic. Thus, for the + phase, the right panel of Fig. 4 applies. Furthermore, in this phase we require $\operatorname{Re}(q)<0$ and the dispersion relation corresponds to the lower (blue) curves of this panel, i.e., $q=q_{3}\left(v_{+}, \Omega\right) \equiv q_{3+}$. In the + phase, the special solution $q_{1}$ must be included only for the stable case $\operatorname{Re} \Omega<0$.

On the other hand, in the - phase we require $\operatorname{Re}(q)>0$, but the outgoing flow may, in principle, be supersonic as well as subsonic. As a consequence, the stability analysis is quite different for weak, strong, or Jouguet deflagrations.

\section{Solution of the perturbation equations}

\section{Supersonic deflagrations}

As we have seen, a supersonic deflagration can be either a strong or a Jouguet solution. Let us consider first the case of strong deflagrations. As already discussed by Landau [12] for a classical gas, strong deflagrations are absolutely unstable, either in the case of a combustion front $(\S 131)$ or a condensation discontinuity $(\S 132)$, due to the fact that such discontinuities are not evolutionary (i.e. there are more free parameters than conditions). According to numerical calculations [27], strong deflagrations are unstable also in the case of a relativistic phase transition. It is interesting to verify this result analytically.

Strong deflagrations are characterized by a supersonic outgoing velocity $v_{-}$, corresponding to the left panel of Fig. 4. For $\operatorname{Re}(\Omega)<0$, we see that all the solutions give $\operatorname{Re}(q)<0$. Therefore, we have no possible modes with $\operatorname{Re}(\Omega)<0$ in the - phase. On the other hand, for $\operatorname{Re}(\Omega)>0$ we have $\operatorname{Re}\left(q_{2}\right)>0, \operatorname{Re}\left(q_{3}\right)>0$, and $\operatorname{Re}\left(q_{1}\right)>0$. We thus have three unstable modes in the - phase. In the + phase, as we have already discussed, we have one unstable mode for $\Omega>0$, namely, $q_{3}$, since $\operatorname{Re}\left(q_{3}\right)<0$ (right panel of Fig. 44). Hence, according to 
Eq. (71), for $z>0$ we must consider a perturbation of the form

$$
\vec{A}(z)=A_{+} \vec{L}_{3+} e^{q_{3+} z}
$$

whereas for $z<0$ we have

$$
\vec{A}(z)=A_{-} \vec{L}_{3-} e^{q_{3-} z}+B \vec{L}_{2-} e^{q_{2-} z}+C \vec{L}_{1-} e^{q_{1-} z} .
$$

Here, the \pm signs in the $q_{i}$ and $\vec{L}_{i}$ mean that these quantities [which are given by (67,69)] must be evaluated at $v_{ \pm}$, respectively. We must impose the junction conditions (46,48) and the surface equation (55) to the fluid variables and the surface deformation $\zeta$. This gives four equations for the five variables $A_{+}, A_{-}, B, C$, and $D$. Such a system of equations has infinite solutions. Hence, the strong deflagration is trivially unstable. Notice that our treatment applies to any of the strong deflagration profiles sketched in Figs. 2 and 3, since we perturbed the fluid near the wall from constant velocity solutions.

As we decrease the velocity and reach the Jouguet point $\left(\left|v_{-}\right|=c_{s-}\right)$, one of the asymptotes becomes vertical and the hyperbola becomes single-valued (central panel of Fig. 4). In this case the solution $q_{3}$ disappears. Thus, we now have only two unstable modes in the - phase, corresponding to $q_{2}$ and the special solution $q_{1}$. Hence, we must set $A_{-}=0$ in Eq. (76) $)$. In the + phase the situation is the same as before (since, for deflagrations, $v_{+}$ is always subsonic), and the unstable mode is given by Eq. (75). As a consequence, the deflagration becomes evolutionary.

As we have discussed in Sec. IIA, for the supersonic Jouguet deflagration the fluid velocity profile develops a tail just behind the wall (right panel of Fig. 3), and our treatment no longer applies. Although it would be interesting to study the stability of this kind of solution, it is quite a difficult task since the stationary velocity profile is a function of $z$ and $t$, namely, $v_{\text {rar }}(z / t)$. Such a study is out of the scope of the present paper and we shall attempt it elsewhere. For the traditional deflagration profile, the Jouguet point just corresponds to the case $v_{w}=c_{s}$, which is the limit between strong and weak deflagrations.

\section{Weak deflagrations}

In the case of weak deflagrations, both velocities $v_{-}$and $v_{+}$are subsonic. We have $\operatorname{Re}\left(q_{2}\right)>0$ and $\operatorname{Re}\left(q_{3}\right)<0$ (right panel of Fig. 4). Hence, we must consider the mode with eigenvalue $q_{3}$ again in the + phase and the mode with eigenvalue $q_{2}$ in the - phase. Besides, for $\operatorname{Re}(\Omega)>0$, we have $\operatorname{Re}\left(q_{1}\right)>0$, and the special solution must be considered in the phase. Thus, in the unstable case, for $z>0$ the function $\vec{A}(z)$ is again of the form (75)), $\vec{A}(z)=A \vec{L}_{3+} e^{q_{3+} z}$, while for $z<0$ we have $\vec{A}(z)=B \vec{L}_{2-} e^{q_{2-} z}+C \vec{L}_{1-} e^{q_{1-} z}$. The junction conditions (46,48), as well as the surface equation (55), require evaluating these functions at the wall position $z=\zeta$. However, to first order in the perturbations, we just evaluate at $z=0$. We thus have, on each side of the interface (omitting a factor $e^{\Omega t+i k x^{\perp}}$ ),

$$
\delta v_{+}=A, \quad \delta p_{+}=-\bar{\gamma}_{+}^{2} \bar{w}_{+} \frac{\Omega+q_{3+} \bar{v}_{+}}{\Omega \bar{v}_{+}+q_{3+}} A, \quad v_{+}^{\perp}=\frac{i k}{\Omega \bar{v}_{+}+q_{3+}} A,
$$

and

$$
\delta v_{-}=B+C, \delta p_{-}=-\bar{\gamma}_{-}^{2} \bar{w}_{-} \frac{\Omega+q_{2-} \bar{v}_{-}}{\Omega \bar{v}_{-}+q_{2-}} B, v_{-}^{\perp}=\frac{i k}{\Omega \bar{v}_{-}+q_{2-}} B+\frac{i q_{1-}}{k} C
$$


These quantities and those related to the corrugation of the wall,

$$
\partial_{0} \zeta=\Omega D, \partial_{\perp} \zeta=i k D, \partial_{0}^{2} \zeta=\Omega^{2} D, \partial_{\perp}^{2} \zeta=-k^{2} D
$$

(omitting again a factor $e^{\Omega t+i k x^{\perp}}$ ), are related by Eqs. (46+48) and (55). We thus have four equations for the four unknowns $A, B, C$ and $D$, i.e., the weak deflagration is evolutionary.

It is interesting to consider also the case of a stable perturbation, $\operatorname{Re}(\Omega)<0$. In this case we have $q_{1}<0$ and the special solution must now be included in the + phase instead of the - phase. The form of the perturbations is similar to that of Eqs. (777) (78), except that the variable $C$ appears in (77) instead of (178). As we shall see, the jump of the special mode from one side of the interface to the other as $\Omega$ changes sign will cause a discontinuity in the wavenumber $k$ as a function of $\Omega$.

Inserting Eqs. (77,79) in Eqs. (46,48,55), we obtain a homogeneous system of linear equations for the constants $A, B, C$ and $D$. Nontrivial solutions exist if the determinant of the matrix associated to this system vanishes. After some manipulations (e.g., multiplying the first column by the factor $Q_{+}$defined below, etc.), this condition can be written in the form

$$
\left|\begin{array}{cccc}
\frac{1}{R_{+}} & \frac{1}{R_{-}} & \frac{\hat{\Omega}}{v_{-} \gamma_{-}^{2}} & -\left(v_{+}-v_{-}\right) \\
v_{-}\left(1+\frac{v_{+} \hat{\Omega}}{R_{+}}\right) & -v_{+}\left(1-\frac{v_{-} \hat{\Omega}}{R_{-}}\right) & v_{+}\left(1+v_{-}^{2}\right) & \hat{\Omega}\left(1-v_{-} v_{+}\right)\left(v_{+}-v_{-}\right) \\
1+\frac{\hat{\Omega}}{v_{+} R_{+}} & -1+\frac{\hat{\Omega}}{v_{-} R_{-}} & 2 & \frac{F_{\mathrm{dr}}}{w_{+}} \frac{1}{v_{+} \gamma_{+}^{2}}\left(\hat{\Omega}^{2}+1\right) k d \\
\frac{\gamma_{s+}^{2}}{2}\left(\gamma_{+} Q_{+}-b_{+} P_{+}\right) & \frac{\gamma_{s-}^{2}}{2}\left(\gamma_{-} Q_{-}-b_{-} P_{-}\right) & -\frac{\gamma_{-}}{2} & -\langle\gamma\rangle \hat{\Omega}+\langle\gamma v\rangle\left(\hat{\Omega}^{2}+1\right) k d
\end{array}\right|=0 .
$$

where $\hat{\Omega} \equiv \Omega / k$, and

$$
R_{ \pm}=\sqrt{\frac{\gamma_{ \pm}^{2}}{\gamma_{s \pm}^{2}}+\frac{\hat{\Omega}^{2}}{c_{s \pm}^{2}}}, \quad P_{ \pm}=v_{ \pm} \mp \frac{\hat{\Omega}}{R_{ \pm}}, \quad Q_{ \pm}=1 \mp \frac{v_{ \pm}}{c_{s \pm}^{2}} \frac{\hat{\Omega}}{R_{ \pm}}
$$

with $\gamma_{s \pm} \equiv 1 / \sqrt{1-v_{ \pm}^{2} / c_{s \pm}^{2}}$. Although the solution is, by symmetry, symmetric in $k$, to obtain these expressions we have assumed $k>0^{7}$. Thus, from now on we are using the notation $k=|k|$.

A solution of Eq. (80) is $\hat{\Omega}=-\gamma_{-} v_{-}$. Indeed, for this value of $\hat{\Omega}$ we have $R_{-}=\gamma_{-}$, $P_{-}=0$, and $Q_{-}=\gamma_{s-}^{-2}$. Hence, the second and third columns of the matrix are proportional, and the determinant vanishes. However, as explained in Ref. [14], this solution is spurious and has no physical significance, as it leads to vanishing values of the variables.

Finding analytical solutions for $\Omega(k)$ from Eq. (80) is a difficult task. Notice, on the other hand, that the wavenumber $k$ appears only in the fourth column, in the matrix elements 34 and 44 . Hence, we can readily find an expression for $k$ as a function of $\hat{\Omega}$,

$$
k d=\frac{\left(v_{+}-v_{-}\right)\left[\operatorname{det}_{14}+\operatorname{det}_{24}\left(1-v_{+} v_{-}\right) \hat{\Omega}\right]-\operatorname{det}_{44}\langle\gamma\rangle \hat{\Omega}}{\left(1+\hat{\Omega}^{2}\right)\left[\left(F_{\mathrm{dr}} / w_{+}\right) \operatorname{det}_{34} /\left(v_{+} \gamma_{+}^{2}\right)-\langle\gamma v\rangle \operatorname{det}_{44}\right]},
$$

\footnotetext{
${ }^{7}$ In particular, we have inserted a factor of $k$ inside a square root in the expressions for $q_{2,3} / k$ to obtain the quantities $R_{ \pm}$.
} 
where $\operatorname{det}_{i j}$ is the determinant of the $3 \times 3$ matrix that results by removing the $i$-th row and the $j$-th column in Eq. (80).

We remark that, for $\operatorname{Re}(\Omega)<0$, we will have a different matrix, since the special mode must be considered in the + phase instead of the - phase. This amounts to changing, in the third column of the matrix, the indices $\pm \leftrightarrow \mp$ and the sign of the first three elements.

\section{STABILITY OF WEAK DEFLAGRATIONS}

We shall now attempt to find all the unstable solutions for a given wavenumber. Notice that, for real $\Omega$, Eq. (82) facilitates to study the general properties of the solution. Moreover, one may obtain a plot of $\Omega$ vs $k$ by just inverting the graph of $k d(\hat{\Omega})$. However, it is not clear form Eq. (82) whether solutions with $\operatorname{Im}(\Omega) \neq 0$ are possible as well.

\section{A. Small velocity limit}

In order to understand the general behavior of the function $\Omega(k)$, it is convenient to consider first non-relativistic velocities, so that we can write down analytical expressions which are rather lengthy in the general case. We shall also use, in this subsection, the approximation of small supercooling, which is consistent with a small wall velocity and avoids considering a particular EOS. Thus, from Eq. (56) and the general driving force (11) we obtain $b_{ \pm} \simeq\langle v\rangle L / F_{\mathrm{dr}}$. Then we may use, for the stationary driving force, the approximation (26),

$$
F_{\mathrm{dr}}=\frac{\langle v\rangle}{v_{+}} L v_{c}^{2}
$$

where $v_{c}=\sqrt{1-T_{+} / T_{c}}$. We thus obtain

$$
b_{ \pm}=v_{+} / v_{c}^{2}
$$

Besides, with these approximations Eq. (5) gives $v_{-}-v_{+}=v_{-} L / w_{+}$plus higher order in $\left(T_{c}-T\right) / T_{c}$. Hence, Eq. (80) becomes

$$
\left|\begin{array}{cccc}
\frac{1}{R_{+}} & \frac{1}{R_{-}} & \frac{\hat{\Omega}}{v_{-}} & v_{-} \bar{L} \\
v_{-}\left(1+\frac{v_{+} \hat{\Omega}}{R_{+}}\right) & -v_{+}\left(1-\frac{v_{-} \hat{\Omega}}{R_{-}}\right) & v_{+} & -\hat{\Omega} v_{-} \bar{L} \\
1+\frac{\hat{\Omega}}{v_{+} R_{+}} & -1+\frac{\hat{\Omega}}{v_{-} R_{-}} & 2 & \left(\hat{\Omega}^{2}+1\right) k d\langle v\rangle \frac{v_{c}^{2}}{v_{+}^{2}} \bar{L} \\
\frac{1}{2}\left(1-\beta_{+}\right) & \frac{1}{2}\left(1-\beta_{-}\right) & -\frac{1}{2} & \left(\hat{\Omega}^{2}+1\right) k d\langle v\rangle-\hat{\Omega}
\end{array}\right|=0
$$

where $\bar{L}=L / w_{+}$, and

$$
R_{ \pm}=\sqrt{1+\frac{\hat{\Omega}^{2}}{c_{s \pm}^{2}}}, \beta_{+}=\frac{v_{+}\left(v_{+}-\frac{\hat{\Omega}}{R_{+}}\right)}{v_{c}^{2}}, \beta_{-}=\frac{v_{+}\left(v_{-}+\frac{\hat{\Omega}}{R_{-}}\right)}{v_{c}^{2}} .
$$

$$
\text { 1. Case }|\hat{\Omega}| \gg\left|v_{ \pm}\right|
$$

Let us first look for solutions of large $|\hat{\Omega}|$. We are interested in the instability case $\operatorname{Re}(\hat{\Omega})>0$, for which we have $R_{ \pm}=\hat{\Omega} / c_{s \pm}\left(1+\mathcal{O}\left(c_{s}^{2} / \hat{\Omega}^{2}\right)\right)$. Hence, to lowest order, the 
parameters appearing in Eq. (85) become

$$
\frac{1}{R_{ \pm}}=\frac{c_{s \pm}}{\hat{\Omega}}, \frac{\hat{\Omega}}{R_{ \pm}}=c_{s \pm}, \beta_{+}=\frac{v_{+}\left(-c_{s+}+v_{+}\right)}{v_{c}^{2}}, \beta_{-}=\frac{v_{+}\left(c_{s-}+v_{-}\right)}{v_{c}^{2}} .
$$

Notice that, in this limit, we have $\beta_{+}>0, \beta_{-}<0$. We can now easily calculate the $3 \times 3$ determinants defined above. The factors of $\hat{\Omega}$ cancel out in $\operatorname{det}_{14}$, whereas $\operatorname{det}_{24}$, $\operatorname{det}_{34}$, and $\operatorname{det}_{44}$ are of the form $\operatorname{det}_{i 4}=\left(\hat{\Omega} / v_{-}\right) \operatorname{det}_{i 4}^{\prime}$, where the $\operatorname{det}_{i 4}^{\prime}$ are determinants of $\hat{\Omega}$-independent $2 \times 2$ matrices. Thus, we have

$$
\Omega d=\frac{1}{\langle v\rangle} \frac{\operatorname{det}_{44}^{\prime}+\bar{L} v_{-} \operatorname{det}_{24}^{\prime}}{\operatorname{det}_{44}^{\prime}-\bar{L} \frac{v_{c}^{2}}{v_{+}^{2}} \operatorname{det}_{34}^{\prime}}
$$

From this expression we see that (in the limit of large $|\Omega / k|$ ) $\Omega$ is be real. More importantly, we can see that the rhs of Eq. (88) is negative, which means that, in fact, we cannot have $|\hat{\Omega}|$ large for $\Omega>0^{8}$. Indeed, consider for simplicity $c_{s+}=c_{s-}$. Using the relation $v_{-}=(1-\bar{L}) v_{+}$and dropping terms $\mathcal{O}\left(v^{2} / c_{s}^{2}\right)$, we obtain

$$
\begin{aligned}
\operatorname{det}_{44}^{\prime} & =2 c_{s}+\bar{L}\left(1+c_{s}^{2}\right)\left|v_{-}\right| \\
v_{-} \operatorname{det}_{24}^{\prime} & =\frac{c_{s}}{2}\left[\frac{\bar{L}}{1-\bar{L}}+\frac{(2-\bar{L})\left|v_{-}\right|\left(c_{s}-\bar{L}\left|v_{-}\right|\right)}{v_{c}^{2}}\right], \\
-\frac{v_{c}^{2}}{v_{+}^{2}} \operatorname{det}_{34}^{\prime} & =\frac{v_{c}^{2}}{v_{+}^{2}} \frac{2-\bar{L}}{2}\left|v_{-}\right|+\frac{\bar{L}\left(c-\bar{L}\left|v_{-}\right|\right)}{2(1-\bar{L})}-\left|v_{-}\right|\left(1+c_{s}^{2}\right),
\end{aligned}
$$

where we have used absolute values to make the signs clearer. We see immediately that $\operatorname{det}_{44}^{\prime}$ and $v_{-} \operatorname{det}_{24}^{\prime}$ are positive (since $\bar{L}<1$ ). On the other hand, in the expression for $-\left(v_{c}^{2} / v_{+}^{2}\right) \operatorname{det}_{34}^{\prime}$, only the last term is negative. However, in Eq. (88) this term cancels with the last term of $\operatorname{det}_{44}^{\prime}$. Hence, since $\langle v\rangle<0$, the rhs of Eq. (88) is negative. We conclude that there are no unstable modes with $|\hat{\Omega}| \gg v_{w}$.

\section{Case $0<|\hat{\Omega}| \lesssim\left|v_{ \pm}\right|$}

Let us consider now the case of smaller $\hat{\Omega}$, up to order $v_{ \pm}$. To linear order in $v_{+}, v_{-}$, and $\hat{\Omega}$, we have $R_{ \pm}=1$, and

$$
\beta_{+}=\frac{v_{+}}{v_{c}^{2}}\left(v_{+}-\hat{\Omega}\right), \beta_{-}=\frac{v_{+}}{v_{c}^{2}}\left(v_{-}+\hat{\Omega}\right) .
$$

In order to compare with the results of Ref. [14], we write down again the determinant in this limit,

$$
\left|\begin{array}{cccc}
1 & 1 & \frac{\hat{\Omega}}{v_{-}} & v_{-} \bar{L} \\
v_{-} & -v_{+} & v_{+} & -v_{-} \hat{\Omega} \bar{L} \\
1+\frac{\hat{\Omega}}{v_{+}} & -1+\frac{\hat{\Omega}}{v_{-}} & 2 & k d \frac{v_{c}^{2}}{v_{+}^{2}}\langle v\rangle \bar{L} \\
\frac{1}{2}\left(1-\beta_{+}\right) & \frac{1}{2}\left(1-\beta_{-}\right) & -\frac{1}{2} & k d\langle v\rangle-\hat{\Omega}
\end{array}\right|=0 .
$$

\footnotetext{
${ }^{8}$ Notice that we are considering the case $\operatorname{Re}(\hat{\Omega})>0$; for $\operatorname{Re}(\hat{\Omega})<0$, the matrix in Eq. 850), as well as the approximations (87), would have different forms.
} 
As expected, the first three rows of the matrix (corresponding to the junction equations for the fluid perturbations) match ${ }^{9}$ those of Ref. [14]. All the differences appear in the forth row (which corresponds to the equation for the interface). Since we considered independent perturbations of variables in each phase, this row is more symmetric in our case. In the case of dependent perturbations, we would replace $\delta v_{-} \rightarrow \delta v_{+}, \delta p_{-} \rightarrow \delta p_{+}$in Eq. (53). We would thus obtain zeros in the elements 42 and 43 (corresponding to the perturbations $\delta v_{-}$ and $\left.\delta p_{-}\right)$and a factor of 2 in the element 41, which would be just given by $1-\beta(1-\eta$ in the notation of [14]).

As we shall see next, for small velocities, the aforementioned differences do not introduce a significant qualitative variation with respect to the results of Ref. [14]. Roughly, the role of that single $\beta$ will be played by the average of $\beta_{+}$and $\beta_{-}$. More important discrepancies will appear for higher velocities. The results will differ significantly also in the case $\Omega \leq 0$, even in the non-relativistic case. Indeed, since the special mode must be considered on either side of the wall according to the sign of $\Omega$, we cannot use Eq. (93) for $\Omega<0$. As a consequence, we shall find a discontinuity in the passage from $\Omega>0$ to $\Omega<0$.

Let us first consider the case $\operatorname{Re}(\Omega)>0$. Besides the aforementioned spurious solution $\hat{\Omega}=-v_{-}$, we obtain a solution of the form

$$
k d=N / D
$$

The expressions for $N$ and $D$ are still rather lengthy, and we shall only write down the case of small $L / w_{+}$. To first order we have

$$
\begin{aligned}
& k d\left[1+\frac{\bar{L}\left(1+\frac{\bar{L}}{2}\right)}{2 \beta}+\frac{\hat{\Omega}}{v_{w}}\right]=\frac{\bar{L}}{2}(1-\beta) \\
& \quad-\left[1+\frac{\bar{L}(1-\bar{L}) \beta}{2}\right] \frac{\hat{\Omega}}{v_{w}}-\left[1+\frac{\bar{L}(1+\beta)}{2}\right]\left[\frac{\hat{\Omega}}{v_{w}}\right]^{2}-\frac{\bar{L} \beta(1+\bar{L})}{2}\left[\frac{\hat{\Omega}}{v_{w}}\right]^{3},
\end{aligned}
$$

where we have defined the parameter

$$
\beta=(1-\bar{L}) \frac{v_{w}^{2}}{v_{c}^{2}} .
$$

In Eqs. (92-96) we have neglected terms of order $v_{w}^{2}$, except in the ratio $v_{w}^{2} / v_{c}^{2}$, since $v_{c}$ may be small. Notice that $v_{c}^{2}=1-T_{+} / T_{c}$ gives a measure of the amount of supercooling. Hence, in the non-relativistic approximation, if we are interested in velocities $v_{w} \sim v_{c}$, the amount of supercooling must be small enough (of order $v_{w}^{2}$ ), i.e., $\left(T_{c}-T_{+}\right) / T_{c}=v_{c}^{2} \sim v_{w}^{2}$.

In order to obtain all the possible values of $\Omega$ (with positive real part), we should invert the relation (95), which amounts to finding the three roots of a cubic polynomial. Notice that the coefficient of the cubic term is in general suppressed with respect to the the linear and quadratic ones. If we neglect this term, we obtain a quadratic equation, with only two roots. The third root of the cubic equation must be large enough to make the cubic term comparable to the quadratic one, i.e., $\hat{\Omega} / v_{w} \sim \bar{L}^{-1}$. This root is in general well beyond the range of validity of the approximation $\hat{\Omega} \lesssim v_{w}$ and must be discarded (in any case, it can

\footnotetext{
${ }^{9}$ Taking into account that $d v_{c}^{2} / v_{+}^{2}=d_{c}$ and the notations $v_{q}=-v_{+}, v_{h}=-v_{-}, \bar{L} \rightarrow \bar{L} / 2$.
} 
be seen that this solution has a negative real part). Let us just assume, for simplicity, that $\bar{L}$ is small enough that we can neglect the cubic term. Then, we have a quadratic equation with real coefficients, and it is trivial to see the structure of the solutions. We have either two complex conjugate roots or two real roots. Since the coefficients of the quadratic and linear terms have the same sign, in the case of complex roots the real part is negative. In the case of real roots, one of them is negative. The other has a smaller absolute value and may be positive or negative, depending on the sign of the $\hat{\Omega}$-independent term. Thus, we have, at most, only one solution with $\operatorname{Re}(\Omega)>0$. This solution has $\operatorname{Im}(\Omega)=0$.

To study this solution, it is convenient to analyze the behavior of $k$ as a function of $\Omega$. We can thus go back to Eq. (95) and consider $\Omega$ real. For $\hat{\Omega}>0$ the lhs of Eq (95) is positive, while most of the terms in the rhs are negative. Indeed, the coefficients of the $\hat{\Omega}$-dependent terms are negative. The $\hat{\Omega}$-independent term is negative for $\beta>1$. In such a case, there is no solution with $\hat{\Omega}>0$ (i.e., we would have unphysical values $k<0$ ), and the deflagration is stable under perturbations of any wavelength. For $\beta<1$, the $\hat{\Omega}$-independent term in the rhs is positive and we have unstable solutions for $\hat{\Omega}$ below a certain value $\hat{\Omega}_{0}$. At $\hat{\Omega}=\hat{\Omega}_{0}$ we have $k=0$ and, as $\hat{\Omega}$ decreases from $\hat{\Omega}_{0}$, the value of $k$ increases. For vanishing $\hat{\Omega}$ we obtain the maximum wavenumber $k_{c}$ for which the perturbation is exponentially unstable.

\section{Analytic approximations}

In the limit of very small $\bar{L}$, the critical value $\beta=1$ is attained for $v_{w}=v_{c}$. This is in agreement with Ref. [14] (in this limit, and for $\hat{\Omega} \rightarrow 0$, we have $\beta_{+}=\beta_{-}=\beta$ ). If $\bar{L}$ is not negligible, the critical velocity is somewhat higher,

$$
v_{\text {crit }}=v_{c} / \sqrt{1-\bar{L}} \approx v_{c}(1+\bar{L} / 2) .
$$

For $v_{w}$ below $v_{\text {crit }}$, we have $\Omega>0$ in the range $0<k<k_{c}$. The value of $k_{c}$ is obtained by setting $\hat{\Omega}=0$ in Eq. (95),

$$
k_{c} d=\frac{\bar{L}}{2} \frac{1-\beta}{1+\left(1+\frac{\bar{L}}{2}\right) \frac{\bar{L}}{2 \beta}} .
$$

This equation shows explicitly the fact that all perturbations are stable for $\beta>1$.

As already mentioned, to obtain the value of $\Omega$ for a given $k$, we should in principle invert the cubic equation (95). In Ref. [14] the corresponding equation is quadratic because the dependence of the parameter $\beta_{+}$on $\Omega$ is neglected. Furthermore, it is argued that the smallness of the velocity (and the fact that $\hat{\Omega} \sim v_{w}$ ) insures that the term quadratic in $\Omega$ is small and can also be neglected, obtaining a linear equation. Notice, however, that $\hat{\Omega} \sim v_{w}$ is not a good argument to neglect any of the terms in the equation. Nevertheless, since the independent term in the rhs of Eq. (95) is always smaller than $\bar{L} / 2$, we have $\hat{\Omega}_{0} / v_{w}<\bar{L} / 2$. Since we always have $\bar{L}<1$ (and often $\ll 1$ ), this is an important constraint. As a consequence, for the unstable range $0<\Omega<\Omega_{0}$, the value of $\hat{\Omega} / v_{w}$ will be, in most cases, small enough to safely neglect the quadratic and cubic terms in Eq. (95). Keeping only the linear terms we obtain

$$
\frac{\hat{\Omega}}{v_{w}}=\frac{\bar{L}}{2} \frac{(1-\beta)\left(1-k / k_{c}\right)}{1+(1-\bar{L}) \frac{\bar{L}}{2} \beta+k d},
$$


which agrees with the result of Ref. [14] for $\bar{L} \ll 1$. Thus, we have

$$
\frac{\hat{\Omega}_{0}}{v_{w}}=\frac{\bar{L}}{2} \frac{1-\beta}{1+(1-\bar{L}) \frac{\bar{L}}{2} \beta}
$$

We see that both $\hat{\Omega} / v_{w}$ and $k d$ are at most of order $\bar{L}$. We plot the function $\hat{\Omega}$ in Fig. 5 ,

Notice that Eq. (99) is valid only for $\hat{\Omega}>0$. Although we are not interested in general in the case $\operatorname{Re}(\Omega)<0$, which is exponentially stable, it is important to consider the limit $\Omega \rightarrow 0^{-}$. As we have seen, for $\operatorname{Re}(\Omega)<0$ the special mode must be considered in front of the wall. This results in a change in the third column of the matrix in Eq. (93), which becomes $\operatorname{col}\left(-\hat{\Omega} / v_{+},-v_{-},-2,-1 / 2\right)$. This will give a jump in $k$ as a function of $\Omega$ (see Fig. 5). Proceeding as before we obtain, for small (and negative) $\hat{\Omega} / v_{w}$,

$$
\frac{\hat{\Omega}}{v_{w}}=\frac{\bar{L}}{2} \frac{\left(1-\beta+\frac{\bar{L}}{2}\right)\left(1-k / k_{c}^{\prime}\right)}{1-(1+\bar{L}) \frac{\bar{L}^{2}}{2} \beta-(1+2 \bar{L}) k d} \quad(\Omega<0),
$$

where the minimum wavenumber for which the perturbation is exponentially stable is given by

$$
k_{c}^{\prime} d=\frac{\bar{L}}{2} \frac{1-\beta+\bar{L}}{1-\left(1+\frac{3 \bar{L}}{2}\right) \frac{\bar{L}}{2 \beta}} .
$$
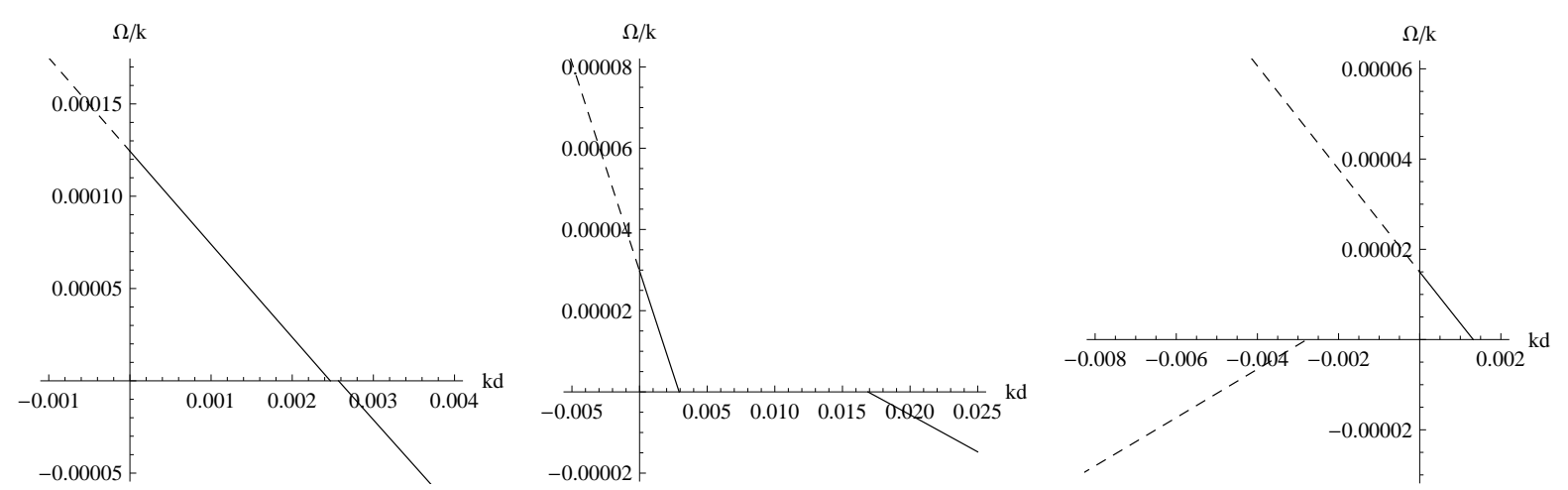

FIG. 5: $\hat{\Omega}$ vs $k d$ in the small $v_{w}$, small $L$ and small $\hat{\Omega} / v_{w}$ approximation, for $\bar{L}=0.01, T_{+} / T_{c}=$ $0.995\left(v_{c} \simeq 0.07\right)$, and $v_{w}=0.05$ (left), 0.007 (center) and 0.003 (right).

Comparing Eq. (101) with Eq. (99), we observe some differences of order $\bar{L} \sim \Delta v / v$ in the expressions for $\Omega>0$ and $\Omega<0$ (due to the changes $v_{ \pm} \leftrightarrow v_{\mp}$ in the third column of the matrix). On the other hand, from Eq. (102) we see that there is also an important sign difference in the denominator of $k_{c}^{\prime}$ with respect to that of $k_{c}$. For $\beta \sim 1$ (i.e., $v_{w} \sim v_{c}$ ), $k_{c}^{\prime}$ will be slightly higher than $k_{c}$. As a consequence, there will be a small gap in the plot of $\Omega$ vs $k$, as can be seen in the left panel of Fig. 5. This gap grows significantly as $v_{w}$ decreases from $v_{c}$ (center panel of Fig. (5) , since $k_{c}^{\prime}$ has a pole at $\beta \approx \sqrt{\bar{L} / 2}$. Hence, for a wall velocity $v_{c}^{\prime} \approx(\bar{L} / 2) v_{c}$ the interval $k_{c}<k<k_{c}^{\prime}$ becomes infinite. Below this velocity, $k_{c}^{\prime}$ takes negative values (right panel of Fig. 5) and the solution with $\Omega<0$ becomes unphysical. There are, in general, more solutions with $\operatorname{Re}(\Omega)<0$, for higher values of $|\Omega|$. We are not interested 
in them, though, since they correspond to stable perturbations. In this case, we have linear stability for $k>k_{c}$.

In the region of $\Omega>0$ our results agree with those of Ref. [14]. In this region, $\hat{\Omega}$ decreases from $\hat{\Omega}_{0}$ to 0 as $k$ increases from 0 to $k_{c}$. Thus, the instability range of $k$ is limited by $k_{c} \lesssim \bar{L} / 2$, whereas $\hat{\Omega}$ is bounded by $\hat{\Omega}_{0} \lesssim v_{w} \bar{L} / 2$. These values are also proportional to $1-v_{w}^{2} / v_{\text {crit }}^{2}$. Therefore, for higher velocities they are even smaller, and stability is recovered at $v_{w}=v_{\text {crit }}$. According to Eqs. (98) and (100), for small velocity we have $k_{c} d \sim v_{w}^{2}$ and $\hat{\Omega} \sim v_{w}$. Hence, stability is also recovered as $v_{w}$ vanishes. This is shown in Fig. 6] (solid lines). For a given velocity, the exponentially unstable wavenumbers are those below the curve of $k_{c}$, whereas the possible values of $\hat{\Omega}$ lie below the curve of $\hat{\Omega}_{0}$. Beyond the critical velocity both $k_{c}$ and $\hat{\Omega}_{0}$ become negative.
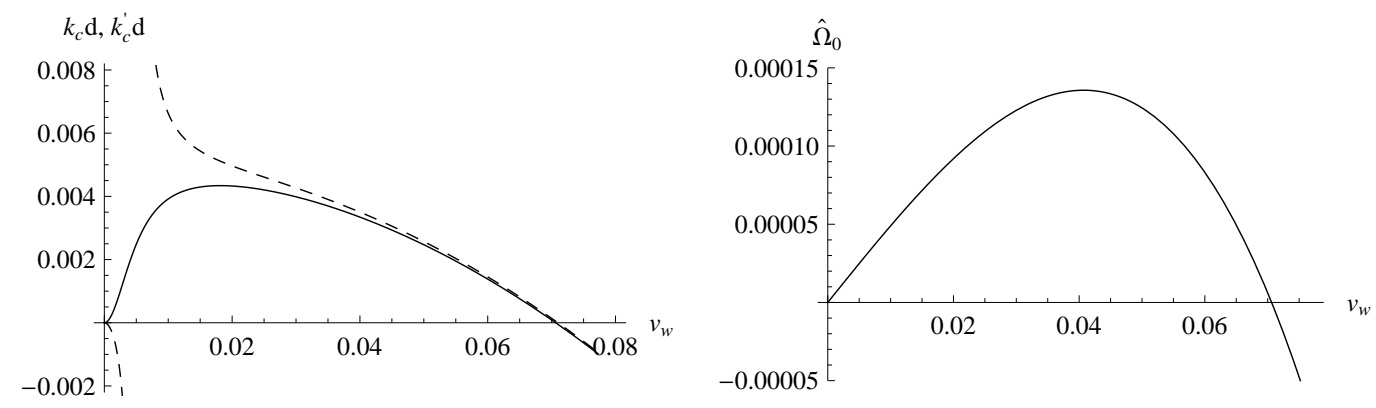

FIG. 6: The values of $k_{c} d$ (solid line), $k_{c}^{\prime} d$ (dashed line), and $\hat{\Omega}_{0}$ as functions of $v_{w}$, for the same set of parameters of Fig. 5 .

For $k \geq k_{c}$, on the other hand, we have significant differences with the results of Ref. [14]. The gap between $k_{c}$ and $k_{c}^{\prime}$ was not observed in that analysis. The reason may be the following. As we have seen, for $\Omega<0$ the only changes in the matrix in Eq. (93) are in the third column. For $\hat{\Omega}=0$, the discontinuity in this column is given by the change $\operatorname{col}\left(0, v_{+}, 2,-1 / 2\right) \rightarrow \operatorname{col}\left(0,-v_{-},-2,-1 / 2\right)$. Since changing the sign of a column does not alter Eq. (93), the above change is equivalent to $\operatorname{col}\left(0, v_{+}, 2,-1 / 2\right) \rightarrow \operatorname{col}\left(0, v_{-}, 2,1 / 2\right)$. In the case of small $\bar{L}$, the change $v_{+} \rightarrow v_{-}$is not relevant. Therefore, the discontinuity is dominated by the change of sign $-1 / 2 \rightarrow 1 / 2$ in the element 43 . However, due to the simplified treatment of Ref. [14], in that work this element is 0 instead of $-1 / 2$.

In principle, at $k=k_{c}, \Omega$ jumps to a value $\Omega<0$ (not shown in Fig. 5 , as it lies beyond the linear approximation). It is not clear, though, from the linear stability analysis, whether there are unstable perturbations or not in the range $k_{c}<k<k_{c}^{\prime}$. For $k<k_{c}$ the perturbations grow exponentially, whereas for $k>k_{c}^{\prime}$ they decay exponentially. In the range between $k_{c}$ and $k_{c}^{\prime}$, one may expect marginal stability with $\Omega=0$. Notice that, as $\Omega \rightarrow 0$, the special solution, which is proportional to $\exp (\Omega z /|v|)$, becomes non-normalizable in either side of the wall, and cannot be included at all in Eqs. (77-78). As a consequence, we will have four equations for the three unknowns $A, B, D$, and the only solution will be the trivial one, $A=B=D=0$. Thus, the approximation of keeping to linear order in perturbations breaks down. It is out of the scope of the present paper to go beyond the linear stability analysis. In any case, in the range of wavenumbers between $k_{c}$ and $k_{c}^{\prime}$, the perturbations will not grow exponentially. For our purposes, it will be enough to assume that, in this range, the possible instabilities would grow more slowly than for $k<k_{c}$. From now on, we shall 
concentrate on the case of $\Omega>0$.

\section{Reheating effects}

The stability of the perturbations depends on the wall velocity and on the temperature $T_{+}$. Notice, however, that the boundary conditions for the fluid fix the temperature beyond the shock front (the nucleation temperature $T_{N}$ ), while $T_{+}$depends on the amount of reheating (see Fig. 2). For a given nucleation temperature $T_{N}$, the temperature $T_{+}$will depend on the wall velocity. As a consequence, $T_{+}$, as well as $v_{w}$, depend on $T_{N}$ and the friction. For the stability analysis it is useful to eliminate the friction and use the wall velocity as a free parameter. However, it is not reasonable to regard $T_{+}$and $v_{w}$ as free independent parameters. In particular, some combinations of $T_{+}$and $v_{w}$ will be unphysical.

For small supercooling (i.e., $T_{N}$ close to $T_{c}$ ), the reheating is given by [28]

$$
\frac{T_{+}}{T_{c}}=\frac{T_{N}}{T_{c}}+\frac{L}{\sqrt{3} w_{+}} v_{w}
$$

In the case $L / w_{+} \ll 1$ this gives $T_{+} \approx T_{N}$. However, a small temperature variation may cause important effects on the wall dynamics. Using Eq. (103) we can write the wall velocity (27) in terms of $T_{N}$,

$$
v_{w}=\frac{w_{+}}{w_{-}} \frac{L\left(1-T_{N} / T_{c}\right)}{\eta_{\mathrm{eff}}}
$$

where we have defined an effective friction coefficient which takes into account the reheating in front of the wall [28]. We have

$$
\frac{\eta_{\mathrm{eff}}}{L}=\frac{\eta}{L}+\frac{L}{\sqrt{3} w_{-}}
$$

Notice that the effects of reheating will depend on the two ratios $\eta / L$ and $L / w_{-} \sim \bar{L}$, whose values are quite unrelated. Thus, the effective friction coefficient $\eta_{\mathrm{eff}}$ may be considerably larger than $\eta$ (even for small $\bar{L}$ ). In particular, for vanishing $\eta$ we will still have a finite effective friction. This hydrodynamic obstruction to the wall motion was discussed more recently in Ref. [32]. Notice that, for $T_{+}$fixed, the velocity would only be bounded by relativity for $\eta \rightarrow 0$ [see Eq.(27)]. In contrast, for $T_{N}$ fixed, according to Eqs. (104,105), the velocity may be bounded by a relatively low value. As a consequence, some velocities will be unreachable, as they would require a negative $\eta$.

Similarly, the velocity $v_{c}$ can be written in terms of $T_{N}$,

$$
v_{c}^{2}=\frac{T_{c}-T_{N}}{T_{c}} \frac{\eta}{\eta_{\mathrm{eff}}}
$$

As we have mentioned, in Ref. [14], the approximation $T_{+}=T_{N}$ was used. Fixing $T_{N}$ and $v_{w}$ instead of $T_{+}$and $v_{w}$, the stability parameter $\beta \approx v_{w}^{2} / v_{c}^{2}$ is enhanced, with respect to that approximation, by a factor of $\eta_{\text {eff }} / \eta$. The ratio

$$
\frac{\eta_{\mathrm{eff}}}{\eta}=1+\frac{1}{\sqrt{3}} \frac{L}{w_{-}} \frac{L}{\eta}
$$

can make a difference if $L / \eta$ is large. Thus, for small velocities (i.e., large $\eta$ ) the approximation $T_{+}=T_{N}$ will not be too bad. Notice, on the other hand, that this is a stabilizing effect. The factor $\eta / \eta_{\text {eff }}$ in Eq. (106) opposes to the increase with $\bar{L}$ in Eq. (97). For high velocities the approximation $T_{+}=T_{N}$ will fail as well as the approximation $T_{-}=T_{+}$. 


\section{B. Arbitrary velocities}

The previous analytic treatment may be extended beyond the limits of the above approximations (e.g., by considering higher orders in $v_{w}, \hat{\Omega}$, or $\bar{L}$ ). However, the equations are rather lengthy to write down here. We have also explored the solutions of Eq. (80) in all the regions of parameter space. We found that most of the qualitative features of $\hat{\Omega}(k)$ hold in the whole range $0<v_{w}<c_{s}$. Thus, the only solution with $\operatorname{Re}(\hat{\Omega})>0$ is real and is always bounded by the value $\hat{\Omega}_{0}$ corresponding to $k=0$. For $k>0$ the value of $\hat{\Omega}$ decreases. In general, $\hat{\Omega}$ vanishes at a finite value $k_{c}$, and we have $\hat{\Omega}_{0} \lesssim v_{w} \bar{L}$ and $k_{c} d \sim \bar{L}$ (there are some exceptions, though; see the next section). Beyond $k_{c}$, there may be a range $k_{c}<k<k_{c}^{\prime}$ of marginal stability. For $k>k_{c}^{\prime}$ the perturbations are exponentially stable. The general behavior of $k_{c}^{\prime}$ is qualitatively similar to that observed analytically. We shall be interested mostly in the case of exponentially unstable perturbations $k<k_{c}{ }^{10}$

Setting $\hat{\Omega}=0$ in Eq. (82) we obtain the critical wavenumber,

$$
k_{c} d=\frac{\Delta v \operatorname{det}_{14}^{0}}{\left(F_{\mathrm{dr}} / w_{+}\right) \operatorname{det}_{34}^{0} /\left(v_{+} \gamma_{+}^{2}\right)-\langle\gamma v\rangle \operatorname{det}_{44}^{0}} .
$$

Setting $k=0$ we obtain, to linear order in $\hat{\Omega}$,

$$
\hat{\Omega}_{0} \simeq \frac{\Delta v \operatorname{det}_{14}^{0}}{\langle\gamma\rangle \operatorname{det}_{44}^{0}-\Delta v\left[\operatorname{det}_{24}^{0}\left(1-v_{+} v_{-}\right)+\operatorname{det}_{14}^{1}\right]} .
$$

We have used the notations $\operatorname{det}_{i j}=\operatorname{det}_{i j}^{0}+\operatorname{det}_{i j}^{1} \hat{\Omega}+\mathcal{O}\left(\hat{\Omega}^{2}\right)$. We write down, as an example, the determinant $\operatorname{det}_{14}^{0}$ (i.e., $\operatorname{det}_{14}$ evaluated at $\hat{\Omega}=0$ ),

$$
\operatorname{det}_{14}^{0}=\Delta v\left[\gamma_{s-}^{2}\left(\gamma_{-}-v_{-} b_{-}\right)-\frac{\gamma_{-}}{2}\right]+\frac{-v_{+}}{\gamma_{-}^{2}}\left\langle\gamma_{s}^{2}(\gamma-v b)\right\rangle
$$

where $b_{ \pm}$are the stability parameters defined in Eq. (56). This determinant dominates the behaviors of $k_{c}$ and $\hat{\Omega}_{0}$. In particular, it can be seen that the denominators in Eqs. (108) and (109) are always positive. Thus, the signs of $k_{c}$ and $\hat{\Omega}_{0}$ depend essentially on the factors $\gamma_{ \pm}-v_{ \pm} b_{ \pm}$in Eq. (110) (notice that $\Delta v$ and $-v_{+}$are positive). Hence, the stability is dominated by the quantities $1-\beta_{ \pm}$, with

$$
\beta_{ \pm}=v_{ \pm} b_{ \pm} / \gamma_{ \pm}
$$

These definitions of $\beta_{ \pm}$are essentially the same as in the previous subsection, but evaluated at $\hat{\Omega}=0$ [cf. Eqs. (86), (92)]. The denominators in Eqs. (108) and (109) become important for $v_{w}$ close to $c_{s-}$. Indeed, in the limit $v_{-} \rightarrow c_{s-}$ we have $\gamma_{s-} \rightarrow \infty$, and $\operatorname{det}_{14}^{0}$ diverges. This divergence is canceled by factors of $\gamma_{s-}$ appearing in the denominators. In any case, $\hat{\Omega}_{0}$ and $k_{c}$ are still dominated by the quantities $1-\beta_{ \pm}$, which appear in all the determinants.

\footnotetext{
${ }^{10}$ A characteristic feature is, thus, that the instability range appears continuously below a critical velocity. Furthermore, $\hat{\Omega}$ grows continuously below $k=k_{c}$, and is in general small. In contrast, in the case of detonations, instabilities generally arise, below a critical velocity, with large values of $\hat{\Omega}$ for all wavenumbers [31].
} 


\section{High velocities and the Jouguet point}

In the non-relativistic, small $\bar{L}$ case, we have $v_{ \pm} \simeq v_{w}, b_{ \pm} \simeq v_{w} / v_{c}^{2}$ [cf. Eq. (84)]. As a consequence, $k_{c}$ and $\hat{\Omega}_{0}$ are proportional to $1-\beta \simeq 1-v_{w}^{2} / v_{c}^{2}$. This simple expression is a consequence of the fact that the driving force is proportional to $v_{c}^{2}=1-T_{+} / T_{c}$ [cf. Eq. (83) $)$. In the general case, it is always possible to define a velocity $v_{c}$ which is proportional to the driving force (hence, $v_{c}$, as well as $F_{\mathrm{dr}}$, will vanish for $T_{-}=T_{+}=T_{c}$ ). Thus, according to Eq. (56), the quantities $b_{ \pm}$will be of the form $\langle\gamma v\rangle / v_{c}^{2}$, and we have

$$
\beta_{ \pm} \approx \frac{\gamma_{ \pm}^{-1}\langle\gamma v\rangle v_{ \pm}}{v_{c}^{2}}
$$

Notice that we have $\beta_{ \pm}>0$. In the case $v_{+} \simeq v_{-}$, we obtain $\beta_{ \pm} \approx v_{w}^{2} / v_{c}^{2}$, and the behavior is similar to the non-relativistic case (namely, $\hat{\Omega}_{0}$ will become negative for a velocity $v_{\text {crit }} \approx v_{c}$ ). However, for large $v_{w}$ we may have a relatively large difference between $v_{+}$and $v_{-}$.

For a deflagration we have $\left|v_{+}\right|<\left|v_{-}\right|=v_{w}$ and, consequently, $\beta_{+}<\beta_{-}$. Therefore, the factor $1-\beta_{-}$vanishes for a certain velocity $v_{w} \gtrsim v_{c}$, but $1-\beta_{+}$remains positive until $v_{w}$ is increased further. As a consequence, the critical velocity $v_{\text {crit }}$ (at which $\hat{\Omega}_{0}$ and $k_{c}$ vanish) will be higher than $v_{c}$. For large $\Delta v$, we may have $v_{\text {crit }}$ close to $c_{s-}$ for relatively low values of $v_{c}$. Moreover, $\left|v_{+}\right|$is bounded by a subsonic value $v_{J}^{\text {def }}$. If $v_{c}$ is higher than this value, then $1-\beta_{+}$may be positive in the whole range $0<v_{w}<c_{s-}$. Then, it may happen that $\hat{\Omega}_{0}$ never becomes negative, i.e., that there is no critical velocity at all. In such a case (which will depend on the amount of supercooling), the deflagration will be unstable for any subsonic velocity. Moreover, as we shall see in the next section, the values of $\hat{\Omega}_{0}$ and $k_{c}$ may become large as $v_{w}$ approaches the speed of sound.

This result is in clear contradiction with Ref. [14], where it is claimed that it is possible to show that, in the limit $v_{w} \rightarrow c_{s}$, the equation for $\Omega$ has no positive roots, for any value of $k$. This discrepancy is, probably, due to the approximations $v_{+}=v_{-}=v_{w}$, $T_{-}=T_{+}$used in [14] for the interface equation. Physically, the stability found for the weak deflagrations in this limit is explained in Ref. [14] by the fact that the result matches with the stability of detonations. However, weak deflagrations never match detonations, as the latter have higher, supersonic velocities $v_{w} \geq v_{J}^{\text {det }}$. Between the speed of sound and the Jouguet detonation velocity $v_{J}^{\text {det }}$, we may have, in principle, either strong deflagrations or Jouguet deflagrations. As we discussed in Sec. III both match the weak deflagration at $v_{w}=c_{s-}$ (i.e., the hydrodynamic solution bifurcates at the Jouguet point). As we have seen, the strong deflagration is unstable, whereas the supersonic Jouguet deflagration is presumably stable in general.

Regardless of the behavior for $v_{w} \rightarrow c_{s-}$, it is easy to show that there cannot be a solution with $\Omega<0$ for $v_{w}=c_{s-}$. Indeed, since $v_{-}=-c_{s-}$, Fig. 4 (central panel) shows that, for $\Omega<0$, all the modes have $q<0$. Thus, we have no mode behind the wall. In front of the wall, we have $v_{+}>-c_{s+}$ (right panel), and we see that there are two modes $\left(q_{1}\right.$ and $\left.q_{2}\right)$ with $q>0$. Applying the linear perturbation analysis, we will have only three unknowns (namely the amplitudes of these two modes and that of the surface deformation) for our four equations (46-48,55). This means that the analysis of linear perturbations breaks down ${ }^{11}$

\footnotetext{
${ }^{11}$ For supersonic Jouguet deflagrations this argument does not apply, since we are considering perturbations from a constant velocity, while this solution has a rarefaction wave immediately after the wall.
} 
for $\Omega<0$.

For $\Omega>0$, in contrast, the calculation is similar to the subsonic case (cf. the center and right panels of Fig. 44), only we must use Eq. (74) for $q_{2}$ instead of Eq. (68). We have checked that the result of such calculation matches the result of the subsonic calculation in the limit $v_{w} \rightarrow c_{s}$.

\section{NUMERICAL RESULTS}

\section{A. The Bag equation of state}

To proceed to the calculation of $\Omega(k)$, we need to consider a concrete equation of state. The simplest phenomenological model for a phase transition is the bag EOS, which consists of radiation and vacuum energy densities (see, e.g., [33]). The pressure in each phase can be written in the form

$$
p_{+}(T)=\frac{a}{3} T^{4}-\frac{L}{4}, \quad p_{-}(T)=\left(\frac{a}{3}-\frac{L}{4 T_{c}^{4}}\right) T^{4} .
$$

The entropy and enthalpy densities can be obtained from $s=d p / d T, w=T s$. This model depends on three parameters, namely, the critical temperature $T_{c}$, the latent heat $L$, and the coefficient $a$. The latter is related to the number of effective massless degrees of freedom in the + phase. The simplicity of the model often allows to obtain analytic results. The speed of sound is the same in both phases $c_{s \pm}=1 / \sqrt{3} \equiv c_{s}$.

The solution for the wall velocity can be obtained from Eq. (10), $\eta\langle\gamma v\rangle=-F_{\mathrm{dr}}$, using the matching conditions (5) 6 ) and the boundary conditions. Since the pressure in both phases is a function of $T^{2}$, it is convenient to use Eq. (12) for the driving force. We obtain

$$
F_{\mathrm{dr}}=\frac{L}{4}\left(1-\frac{T_{-}^{2} T_{+}^{2}}{T_{c}^{4}}\right) .
$$

The matching conditions give the relations

$$
\begin{gathered}
\frac{T_{-}^{2}}{T_{+}^{2}}=\sqrt{\frac{v_{+} \gamma_{+}^{2}}{v_{-} \gamma_{-}^{2}(1-\bar{L})}}, \\
v_{+}=\frac{1}{1+\alpha_{+}}\left[\frac{1}{6 v_{-}}+\frac{v_{-}}{2} \pm \sqrt{\left(\frac{1}{6 v_{-}}+\frac{v_{-}}{2}\right)^{2}+\alpha_{+}^{2}+\frac{2}{3} \alpha_{+}-\frac{1}{3}}\right],
\end{gathered}
$$

where

$$
\bar{L} \equiv \frac{L}{4 a T_{c}^{4} / 3}=\frac{L}{w_{+}\left(T_{c}\right)}
$$

and

$$
\alpha_{+} \equiv \frac{L}{4 a T_{+}^{4}}=\frac{\bar{L}}{3} \frac{T_{c}^{4}}{T_{+}^{4}} .
$$

The + sign in Eq. (116) corresponds to detonations, and the - sign to deflagrations. For weak deflagrations, we have $v_{-}=-v_{w}$, and the reheating temperature $T_{+}$is related to the 
nucleation temperature $T_{N}$ by

$$
\frac{\sqrt{3}\left(T_{+}^{4}-T_{N}^{4}\right)}{\sqrt{\left(3 T_{+}^{4}+T_{N}^{4}\right)\left(3 T_{N}^{4}+T_{+}^{4}\right)}}=\frac{v_{+}-v_{-}}{1-v_{+} v_{-}} .
$$

We define the velocity $v_{c}$ by

$$
v_{c}^{2} \equiv \frac{1}{4}\left(1-\frac{T_{-}^{2} T_{+}^{2}}{T_{c}^{4}}\right)
$$

so that we have $F_{\mathrm{dr}}=L v_{c}^{2}$. The velocity $v_{c}$ is symmetric in $T_{+}$and $T_{-}$. For small supercooling we have $v_{c}^{2} \simeq \frac{1}{2}\left(1-T_{+} T_{-} / T_{c}^{2}\right) \simeq 1-\sqrt{T_{+} T_{-}} / T_{c}$. For small latent heat we have $T_{-} \simeq T_{+}$ and we recover the definition $v_{c}^{2}=1-T_{+} / T_{c}$. From Eqs. (56) and (114) we see that the coefficients $b_{ \pm}$are equal,

$$
b_{ \pm}=\frac{\langle\gamma v\rangle}{v_{c}^{2}} \frac{T_{+}^{2} T_{-}^{2}}{T_{c}^{4}}
$$

and the quantities $\beta_{ \pm}$defined in (111) are given by

$$
\beta_{ \pm}=\frac{\gamma_{ \pm}^{-1}\langle\gamma v\rangle v_{ \pm}}{v_{c}^{2}} \frac{T_{+}^{2} T_{-}^{2}}{T_{c}^{4}}
$$

For small supercooling and small latent heat, we have $\beta_{ \pm} \simeq v_{w}^{2} / v_{c}^{2}$, and we have a critical velocity $v_{\text {crit }}=v_{c}$. In the general case, the temperature ratios in Eq. (122) enhance the value of $v_{\text {crit }}$ with respect to $v_{c}$. Furthermore, the fact that $\left|v_{+}\right|<\left|v_{-}\right|$implies that $\beta_{+}<\beta_{-}$, as already discussed.

\section{B. Stability of deflagrations}

In Fig. 7 we plot the set of real solutions for $\hat{\Omega}$ as a function of $k d$ (the right panel zooms at small $k d)$. For $\operatorname{Re}(\hat{\Omega})>0$, we have found no other solutions (neither real nor complex). We considered values of the parameters similar to those considered in Ref. [14], namely, a very small value of the latent heat $(\bar{L}=0.01)$ and a value of $T_{+}$very close to $T_{c}$, which gives a small ${ }^{12}$ critical velocity, $v_{\text {crit }} \simeq v_{c} \simeq 0.07$. We have chosen a wall velocity below the critical one, $v_{w}=0.05$, so that there is a range of unstable wavenumbers. In the right panel we have also plotted the approximations (99) and (101). Notice that these do not match the exact solution even for vanishing $\hat{\Omega}$. This is because the approximations are linear not only in $\hat{\Omega}$, but also in the parameters $v_{w}, v_{c}$ and $\bar{L}$.

Changing the values of the parameters, the behavior is qualitatively similar (as we already discussed analytically). Essentially, the effect will be a variation of the points where the curves cut the axes, i.e., of the parameters $\hat{\Omega}_{0}, k_{c}$, and $k_{c}^{\prime}$ (see Fig. 5). In Fig. 8, the two parameters which characterize the instability (namely, $k_{c}$ and $\hat{\Omega}_{0}$ ) are plotted as functions of the wall velocity. The lower curve corresponds to the parameters of Fig. 7 and is well approximated by the non-relativistic approximation shown in Fig. 6. The other curves in Fig. 7 correspond to higher values of the latent heat. As already seen with analytic

\footnotetext{
$\overline{12}$ See the discussion below Eq. (96).
} 

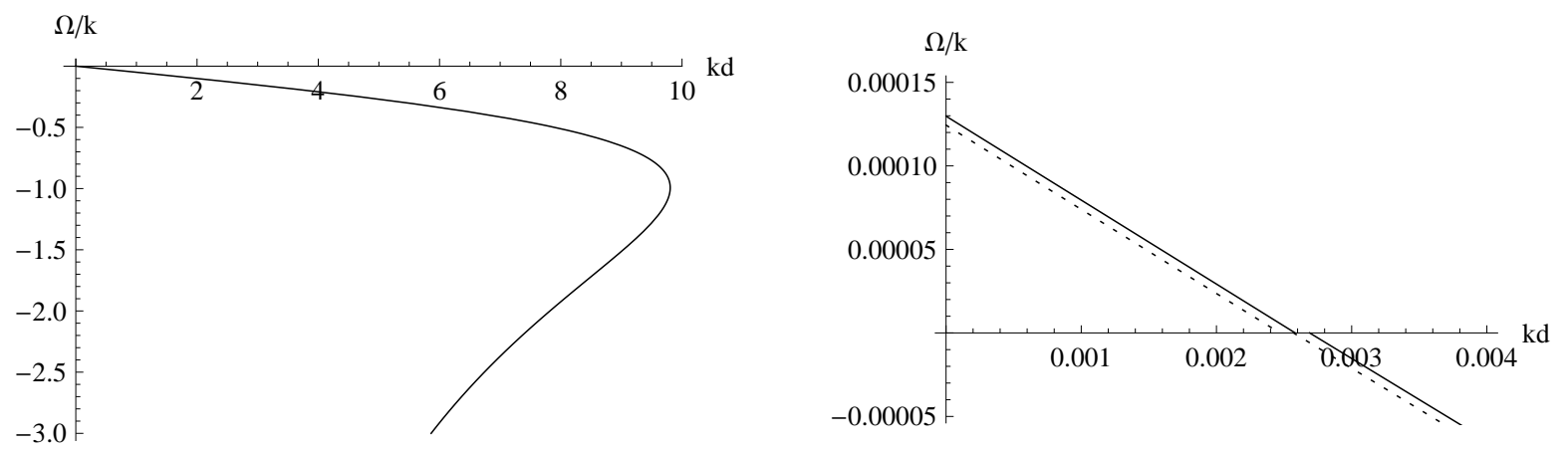

FIG. 7: $\hat{\Omega}$ vs $k d$ for $\bar{L}=0.01, T_{+} / T_{c}=0.995$, and $v_{w}=0.05$. The right panel shows also the linear approximations (dotted line).
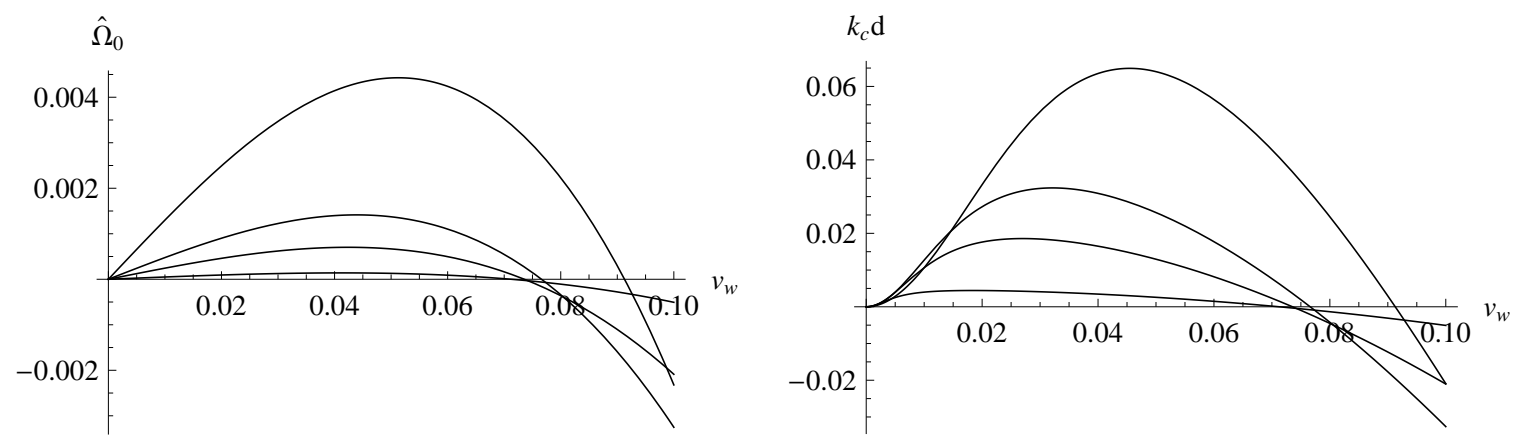

FIG. 8: The maximum value of $\Omega / k$ (corresponding to $k=0$ ) and the maximum unstable wavenumber $k_{c}$ (corresponding to $\hat{\Omega} \rightarrow 0^{+}$), as functions of the wall velocity, for $T_{+} / T_{c}=0.995$ and, from bottom to top, $\bar{L}=0.01,0.05,0.1$ and 0.3 .

approximations, the critical velocity increases with the latent heat. Notice, however, that this effect is quite small. Although $k_{c}$ and $\Omega_{0}$ are proportional to $\bar{L}$, the critical velocity hardly varies with $\bar{L}$.

The dependence on the amount of supercooling is more important (see Fig. 9). For small supercooling we have $v_{\text {crit }} \simeq v_{c} \simeq \sqrt{1-T_{+} / T_{c}}$. As we increase the amount of supercooling, we observe that $v_{\text {crit }}$ grows more quickly than $v_{c}$, as predicted in the previous section. Thus, e.g., for $T_{+} / T_{c}=0.92$, we have $v_{c} \simeq 0.28$ while $v_{\text {crit }} \simeq 0.38$. We see that this behavior becomes critical for a value of $T_{+} / T_{c} \simeq 0.9$, which corresponds to $v_{c} \simeq 0.31$, while the critical velocity reaches a value $v_{\text {crit }} \simeq 0.5$. Here, a second critical velocity appears, beyond which $\hat{\Omega}_{0}$ and $k_{c}$ become positive again. Increasing slightly the amount of supercooling, the critical velocity ceases to exist and the deflagration is unstable in all the range $0<v_{w}<c_{s}$.

Notice that, as we increase the supercooling, the non-relativistic regime of $v_{w}$ does not suffer qualitative modifications, while the relativistic regime changes considerably. This happens because the difference $\Delta v=v_{+}-v_{-}$grows with $v_{w}$. As discussed in Sec. IVB, a high value of $\Delta v$ prevents $\hat{\Omega}_{0}$ and $k_{c}$ to become negative. This effect is so important that, beyond a certain amount of supercooling, $v_{\text {crit }}$ disappears before reaching $c_{s}$. Moreover, the 

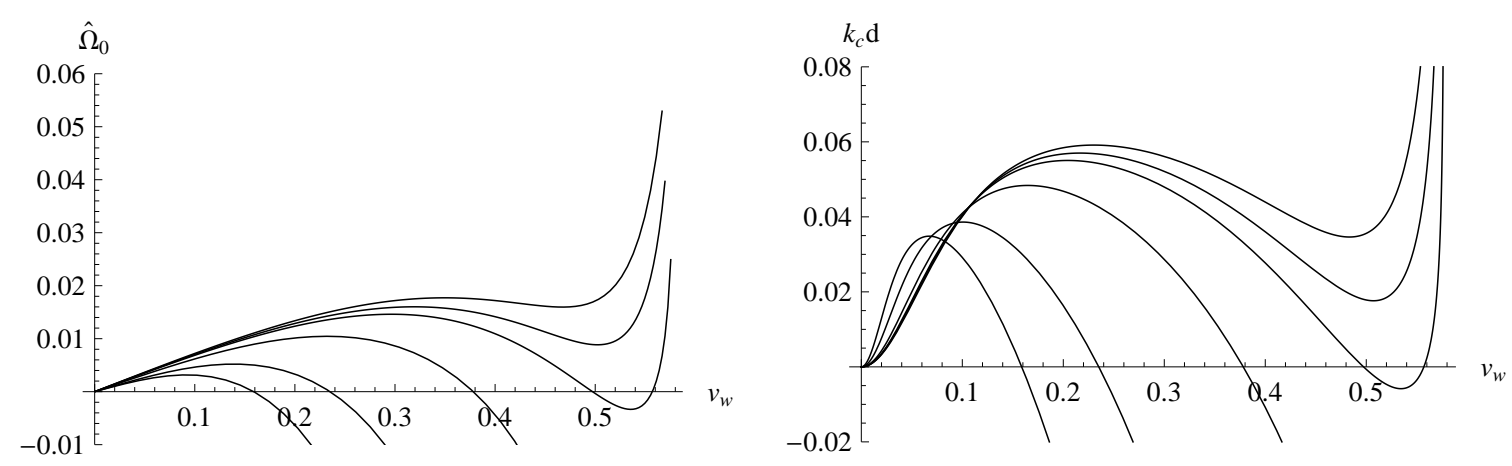

FIG. 9: The values of $\hat{\Omega}_{0}$ and $k_{c} d$ as functions of the wall velocity, for $\bar{L}=0.1$ and, from bottom to top, $T_{+} / T_{c}=0.98,0.96,0.92,0.9,0.895$ and 0.89 .

value of $\hat{\Omega}_{0}$ at $v_{w}=c_{s}$ begins to grow very quickly, and the value of $k_{c}$ diverges.

\section{Reheating effects}

As already discussed, it is important to notice that $T_{+}$is not the nucleation temperature, and should not be considered as a free parameter. The reheating in front of the wall increases with the wall velocity. Hence, if we fix $T_{N}$ and increase $v_{w}$, the temperature $T_{+}$will get closer to $T_{c}$, reducing the value of $v_{c}$.

To see the importance of this effect, let us consider the wall velocity as a function of the friction coefficient $\eta$. In the left panel of Fig. 10 we show the relation between velocity and friction for fixed $T_{+}$, corresponding to some of the curves of Fig. 9. The dots indicate the critical velocity. Thus, below the dots the deflagration is unstable under long wavelength perturbations $\left(\lambda>1 / k_{c}\right)$. For the upper curve, the critical velocity does not exist and deflagrations of any velocity have instabilities. Notice that (fixing $T_{+}$) deflagrations do not exist for small enough $\eta$. Besides, for some values of the friction, there are two possible wall velocities.

In the right panel of Fig. 10, we fix instead the value of $T_{N}$ (to the same values given previously to $T_{+}$). For small $v_{w}$ (large $\eta$ ), the results are similar, indicating that $T_{+} \simeq T_{N}$. However, for higher $v_{w}$ (smaller $\eta$ ) it becomes apparent that the velocity is smaller than in the left panel. This is because $T_{+}$is closer to $T_{c}$ and hydrodynamics acts as an effective friction. In particular, for small supercooling (lower curves), we see that the deflagration is always subsonic, even for $\eta=0$. This means that, depending on the parameters, not any velocity will be physically reachable. This fact may be missed when we consider $v_{w}$ instead of $\eta$ as a free parameter.

In Fig. 11 we plot again the values of $\hat{\Omega}_{0}$ and $k_{c}$, this time fixing $T_{N}$ and taking into account the reheating. We considered some of the previous values of $T_{N}$, as well as higher amounts of supercooling. We see that the behavior is softened with respect to Fig. 9, The critical velocity now grows more slowly, and reaches $v_{\text {crit }}=c_{s}$ at a temperature $T_{N} \simeq 0.775$. As the amount of supercooling is increased further, wall velocities close to the speed of sound become more and more unstable.

Thus, we may have two different situations, depending on the amount of supercooling. If the latter is small enough, we have $v_{\text {crit }}<c_{s}$ and the deflagration is stable in the range 

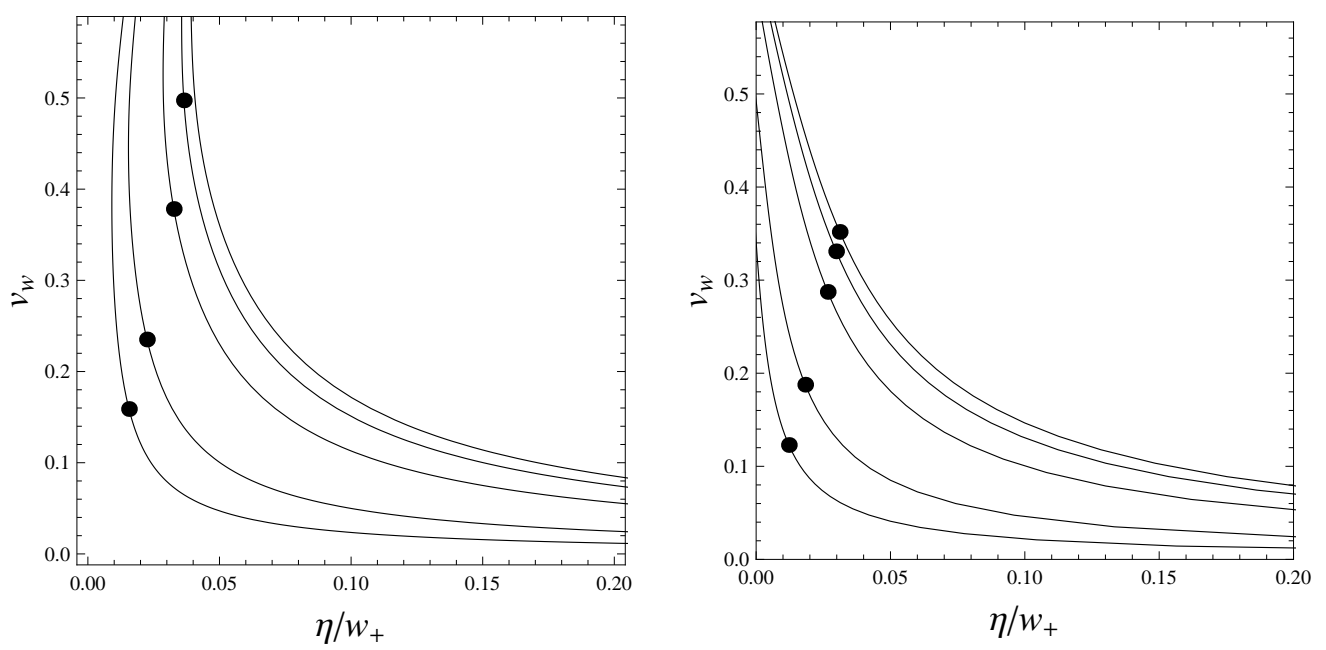

FIG. 10: The wall velocity as a function of the friction, for $\bar{L}=0.1$. In the left panel $T_{+}$is fixed. In the right panel, $T_{N}$ is fixed. The values of $T_{+} / T_{c}$ and $T_{N} / T_{c}$ are, from bottom to top, 0.98 , $0.96,0.92,0.9$ and 0.89 .
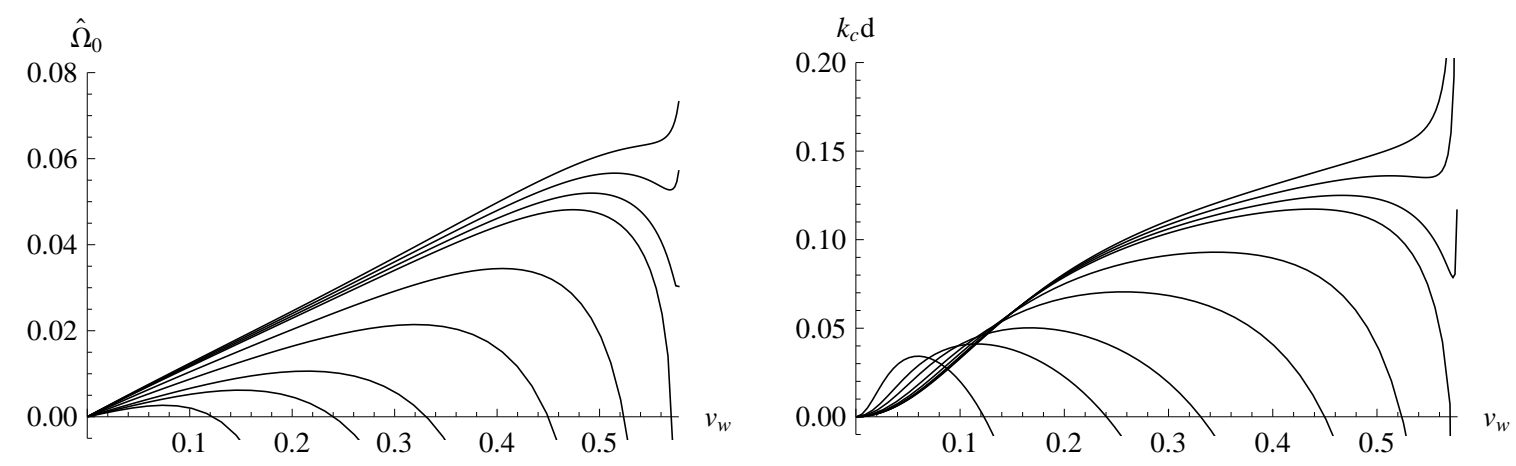

FIG. 11: The values of $\hat{\Omega}_{0}$ and $k_{c} d$ as functions of the wall velocity, for $\bar{L}=0.1$ and different values of the nucleation temperature. From bottom to top, we have $T_{N} / T_{c}=0.98,0.94,0.9,0.84,0.8$, $0.775,0.77,0.765$ and 0.76 .

$v_{\text {crit }}<v_{w}<c_{s}$. Since the weak deflagration matches the supersonic Jouguet deflagration at $v_{w}=c_{s}$, this may be an indication of the stability of the latter. On the other hand, after the critical velocity reaches the value $c_{s}$, the situation is inverted; velocities close to $c_{s}$ are the most unstable ones. This instability may be an indication of the instability of the supersonic Jouguet deflagration. This result suggests that, under the conditions which give weak deflagration velocities close to $c_{s}$ (a high amount of supercooling and a low friction), another solution is hydrodynamically favored, namely, the weak detonation.

\section{EFFECTS OF THE INSTABILITY}

In this section we shall consider the effects of the instability on the dynamics of a cosmological phase transition. 


\section{A. Bubble growth and surface corrugation}

As mentioned in Sec. IIIA, bubbles nucleate with an initial radius which is of the order of the scale $d$. Their walls accelerate during a time which is also of order $d$, after which they reach a terminal velocity. The scale $d$ is in general much smaller than the final bubble radius or the duration of the phase transition (the latter two are related by $R_{f} \sim v_{w} \Delta t$ ). Indeed, although both $d$ and $\Delta t$ depend on the non-trivial dynamics of the phase transition, the former is determined by forces which are not related to the expansion rate of the universe, $H$, whereas the latter will be a fraction of the age of the Universe, $t \sim H^{-1}$. Roughly, we have $d \sim T^{-1}$ and $\Delta t \sim M_{P} / T^{2}$, where $M_{P}$ is the Planck mass. Hence, we have $d / \Delta t \sim T / M_{P}$, which is, for most phase transitions, many orders of magnitude less than 1 . Therefore, the terminal velocity is reached almost immediately. During most of its growth, the bubble will be in a stationary state, unless the growth becomes unstable under small perturbations ${ }^{13}$.

Let us assume that we have instabilities, and consider their growth. We shall concentrate on exponentially unstable perturbations (i.e., $\Omega>0$ ). We remark, though, that there is a velocity $v_{c}^{\prime}$ around which perturbations of any wavelength are marginally unstable (see the discussion around Figs. 5 and 6).

In most cases, the behavior of the instability can be described (at least qualitatively) by the analytic approximations derived Sec. IV, except in the limit in which both $v_{\text {crit }}$ and $v_{w}$ are very close to $c_{s}$, where the behavior departs significantly from these approximations. For $v_{w}<v_{\text {crit }}$, the essential features of the instability are already present in Link's result, namely, that $\Omega$ is of order $\bar{L}$ and proportional to the wall velocity, as well as the dependence $\Omega(k) \propto k\left(k-k_{c}\right)$ [see Eq. (14)]. Thus, for the purposes of the present discussion, we shall use the approximations (98, 100), to lowest order in $\bar{L}$. In particular, we have

$$
k_{c} d \simeq \frac{\beta \bar{L} / 2}{\beta+\bar{L} / 2}(1-\beta) .
$$

In most cases the parameter $\bar{L}$ will be small. Hence, for $\beta \sim 1$ we have

$$
k_{c} d \simeq \frac{\bar{L}}{2}(1-\beta) \quad(\beta \sim 1)
$$

However, in some cases we may have a very small velocity (e.g., due to a significant reheating during bubble expansion). In such a case we have

$$
k_{c} d \simeq \beta \quad(\beta \ll 1) .
$$

In any case, we may neglect $k d$ in the denominator of Eq. (99) and write

$$
\Omega / k \simeq \hat{\Omega}_{0}\left(1-k / k_{c}\right)
$$

with

$$
\hat{\Omega}_{0} \simeq \bar{L} v_{w}(1-\beta) / 2
$$

\footnotetext{
${ }^{13}$ In Ref. [14] the results of the stability analysis were applied to the acceleration stage (although they were derived for the stationary motion). The conclusion was that, since the terminal velocity is reached when the bubble size is $\sim d<\lambda_{c}$, the growth is not destabilized during this stage. We shall assume that the wall reaches the stationary state before it can become unstable.
} 
Thus, perturbations above the critical wavelength $\lambda_{c}=1 / k_{c}$ are unstable. Notice, though, that stability is recovered for $\lambda \rightarrow \infty$. Indeed, the finite value of $\hat{\Omega}_{0}$ implies that $\Omega$ vanishes at $k=0$ (see Fig. 12, left panel). The stability of the zero mode may be understood as follows. This perturbation corresponds to acceleration of the wall without corrugation. However, we know that the uncorrugated wall has already undergone an acceleration stage and has reached a terminal velocity, ending in this stationary state. In a way, the stability for $k=0$ just confirms the existence of such a stationary state. On the other hand, if we allow the wall to be deformed, instabilities arise. In this case, the corrugation introduces a length scale, and the relevant quantity will not be the value of $\Omega$ but the dimensionless combination $\Omega / k$. The latter is a velocity and in principle should be contrasted with $v_{w}$. Thus, an important parameter will be $\hat{\Omega}_{0} / v_{w} \sim k_{c} d \lesssim \bar{L} / 2$ (see Fig. 12, right panel).
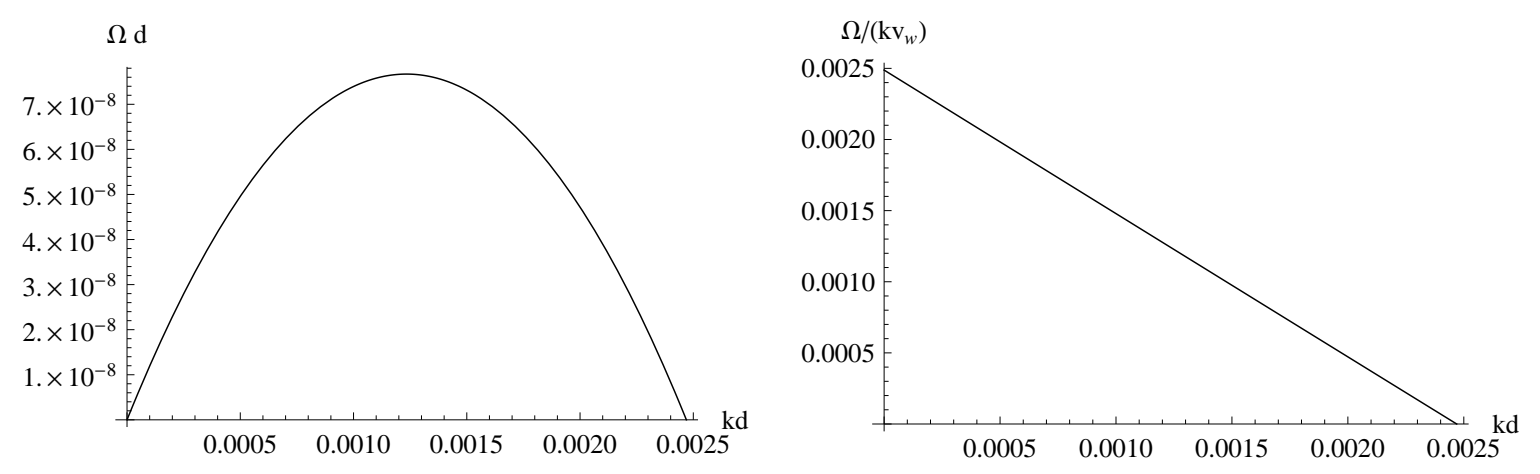

FIG. 12: The values of $\Omega$ and of the dimensionless quantity $\Omega /\left(k v_{w}\right)$ as functions of $k$, for the same parameters of Fig. 5 ,

As can be seen in the left panel of Fig. 12, there is a mode with maximum growth rate. For the approximation (126), the wavenumber of this mode is $k=k_{c} / 2$, and the growth rate is $\Omega_{\max }=\hat{\Omega}_{0} k_{c} / 4$. We may obtain a stability criterion by considering this mode, which has the shortest growth time $\tau \sim \Omega_{\max }^{-1}[13,34]$. Notice that bubbles have a finite size $R_{b}$ and, thus, cannot admit corrugations of arbitrary scales. At the beginning, bubbles are very small, $R_{i} \sim d$. From the equations above, we see that the critical wavelength is higher than that, $\lambda_{c}>2 d / \bar{L}$. Hence, physical perturbations (i.e., those with $\lambda<R_{b}$ ) will be stable until bubbles reach a size $R_{b}>\lambda_{c}$. Besides, the time available for an instability to grow is bounded by the duration of the phase transition, $\Delta t \approx R_{f} / v_{w}$. The mode with the shortest growth time $\Omega_{\max }^{-1}$ will be able to develop if $R_{f}$ is larger than the corresponding wavelength $2 / k_{c}$, and if $\Delta t>\Omega_{\max }^{-1}$. The two conditions are thus $R_{f}>2 / k_{c}$ and $\Delta t>4 /\left(\hat{\Omega}_{0} k_{c}\right)$. The latter condition (which implies the former) gives

$$
R_{f}>\frac{4 / k_{c}}{\hat{\Omega}_{0} / v_{w}} .
$$

This instability criterion can be improved, if we notice that modes with longer growth times may have, on the other hand, more time available to develop. Indeed, a perturbation with wavenumber $k$ can only be formed after the bubble reaches a size $\lambda=1 / k$. The time elapsed since $\lambda$ "enters" the bubble size until the bubble reaches a larger size $R_{b}$ is given by $\Delta t_{k}=\left(R_{b}-1 / k\right) / v_{w}$. On the other hand, the perturbation (if unstable) grows in a time 
$\tau \sim \Omega^{-1}$. Therefore, the mode $k$ will become dynamically important when $R_{b}$ is such that $\Omega \Delta t_{k} \gtrsim 1$. Using the approximation (126) we obtain

$$
\Omega \Delta t_{k}=\frac{\hat{\Omega}_{0}}{v_{w}}\left(1-\frac{\lambda_{c}}{\lambda}\right)\left(\frac{R_{b}}{\lambda}-1\right) .
$$

This equation takes into account the fact that perturbations are linearly unstable only in the range $\lambda_{c}<\lambda<R_{b}$. The first two factors in Eq. (129) are smaller than 1. Moreover, we have in general $\hat{\Omega}_{0} / v_{w} \ll 1$. However, the last factor may be large, depending on the final bubble size.

For a given $R_{b}$, the dynamically most relevant perturbation is now given by the maximum of $\Omega \Delta t_{k}$,

$$
\left(\Omega \Delta t_{k}\right)_{\max }=\frac{\hat{\Omega}_{0}}{v_{w}} \frac{\left(R_{b} k_{c}-1\right)^{2}}{4 k_{c} R_{b}},
$$

which is attained for a wavenumber

$$
k=\frac{1}{2}\left(\frac{1}{R_{b}}+k_{c}\right) .
$$

This perturbation will be important if $\left(\Omega \Delta t_{k}\right)_{\max } \gtrsim 1$. If we apply this criterion to the final bubble size, for which we have $R_{f} \gg 1 / k_{c}$, we obtain Eq. (128). On the other hand, when the bubble size is still comparable to the critical wavelength, the criterion says that no instability is important. The instabilities become important once the bubble reaches the size

$$
R_{b}^{\text {inst }}=\frac{1 / k_{c}}{\hat{\Omega}_{0} / v_{w}}\left[1+\sqrt{1+\hat{\Omega}_{0} / v_{w}}\right]^{2} .
$$

Since in general $\hat{\Omega}_{0} / v_{w}$ is small, we have $R_{b}^{\text {inst }} \approx\left(4 / k_{c}\right) /\left(\hat{\Omega}_{0} / v_{w}\right)$, as in Eq. (128).

The parameters $\hat{\Omega}_{0}$ and $k_{c}$ are not independent. For $\beta \sim 1$ we can use the approximation (124), which gives $k_{c} d \approx \hat{\Omega}_{0} / v_{w} \approx(1-\beta) \bar{L} / 2$. Thus, we have

$$
R_{b}^{\text {inst }} \simeq\left[\frac{4 / \bar{L}}{1-\left(v_{w} / v_{\text {crit }}\right)^{2}}\right]^{2} d \quad(\beta \sim 1) .
$$

For $v_{w} \rightarrow v_{\text {crit }}$, Eq. (133) diverges, meaning that the instabilities need infinite time to develop. On the other hand, for $v_{w} \rightarrow 0$ we must use the approximation (125). Although $\hat{\Omega}_{0} / v_{w}$ does not vanish in this limit, $k_{c}$ does, and we have

$$
R_{b}^{\text {inst }} \simeq \frac{8}{\bar{L}} \frac{v_{\text {crit }}^{2} d}{v_{w}^{2}} \simeq \frac{8}{\bar{L}} \frac{\sigma / L}{v_{w}^{2}} \quad(\beta \ll 1),
$$

where we have used the relations $d=\sigma / F_{\mathrm{dr}}$ and $F_{\mathrm{dr}} \simeq L v_{c}^{2}$ to make explicit the fact that $v_{\text {crit }}^{2} d$ does not depend on the temperature $T_{N}$. In most cases, we will have $\beta \sim 1$. Therefore, Eq. (133) can be used to determine the bubble size at which the instabilities become important. Roughly, we have $R_{b}^{\text {inst }} / d \sim(4 / \bar{L})^{2}$ (unless there is a fine tuning so that $v_{w} \simeq v_{\text {crit }}$ ). Thus, $R_{b}^{\text {inst }}$ will be in general quite higher than the initial bubble size $d$. On the other hand, as we have mentioned, the final bubble size $R_{f}$ will be a fraction of the Hubble radius $H^{-1}$, which is many orders of magnitude larger than $d$ and, in general, much larger than $R_{b}^{\text {inst }}$ as 
well. Notice also that, as can be seen from Eq. (132) we have, in general, $R_{b}^{\text {inst }} \gg 1 / k_{c}$. As a consequence, the dynamically most relevant wavenumber will be, according to Eq. (131), close to $k \simeq k_{c} / 2$.

As pointed out in Ref. [34], the stability may be recovered due to reheating. Indeed, once the shock fronts (which precede the phase transition fronts at a speed $v_{\text {sh }} \simeq c_{s}$ ) meet, they may reheat the space back to a temperature $T_{r}$ very close to $T_{c}$. In such a case, a phase equilibrium stage begins, during which the regions of stable phase can grow only at the rate at which the adiabatic expansion takes the latent heat away [3, 4, 41]. From the viewpoint of the instabilities, the effect would be, roughly, to change the boundary condition for the temperature from $T_{N}$ to $T_{r}$. As a consequence, both velocities $v_{c}$ and $v_{w}$ will decrease significantly. It is not clear which will be the value of $\beta$. Nevertheless, since $d \simeq \sigma /\left(L v_{c}^{2}\right)$, we see that either of Eqs. (133) and (134) will give a large value of $R_{b}^{\text {inst }}\left(T_{r}\right)$. To appreciate the importance of the change of the scale $d$ after reheating, we notice that the new wall velocity is proportional to the expansion rate, $v_{w} \sim R_{b} H / \bar{L}$ [4, 35]. Thus, Eq. (134) gives an enhancement $\sim\left(H^{-1} / R_{b}\right)^{2}$, which ensures the stability of the deflagration. Notice that the time available for the instabilities to grow is the same as before $\left(\Delta t \sim R_{f}\right)$, since the spacing between bubble centers is given by $R_{f}$ and the reheating will occur after a time of order $\Delta t=R_{f} / v_{\mathrm{sh}}$. The only difference is that, if a phase equilibrium stage is reached, the total duration of the phase transition will be longer than $\Delta t$.

In summary, the time and length scales are the following. After a bubble nucleates with size $\sim d$, it reaches the stationary motion in a time $\sim d$. The instabilities (provided that $v_{w}<v_{\text {crit }}$ ) become dynamically relevant much later, after a time $\sim(4 / \bar{L})^{2} d$. In general, though, there will be ample time for this to happen during bubble expansion, since the final bubble size is still much larger $\left(R_{f} \sim\right.$ a fraction of $\left.H^{-1}\right)$. After a time $\sim R_{f}$, the phase transition may end, or it may enter a phase-equilibrium stage during which stable growth is recovered. The dynamically relevant unstable modes are those of wavelengths $\sim 4 d / \bar{L}$. This is generally much shorter than the average bubble size $R_{f}$ by the end of the phase transition.

\section{B. Deflagrations vs detonations}

For strong supercooling or small friction, the stationary solution is in general a detonation with high velocity. On the contrary, for $T_{N}$ very close to $T_{c}$ or large $\eta$ we generally have weak deflagrations with small velocities. Between these two extremes, we may have coexistence of subsonic and supersonic solutions (see e.g. [25, 28]). In such a case, the question arises of which one will be realized during the phase transition, and of whether this can be elucidated by the analysis of instabilities. These issues were discussed previously from a different approach, namely, in the context of a numerical investigation of Eqs. (1-2) in a grid [27]. Instead of using an approximation for the interface, the field configuration $\phi(\mathbf{x}, t)$ was considered together with the fluid profile. Thus, the dynamical evolution of a phase transition front was studied, from the initial acceleration period to the collision between two bubbles.

Regarding the coexistence of deflagrations and detonations, it was found in [27] that, in the cases in which either of the stationary solutions is possible, it is the detonation the one which is realized during bubble expansion. Nevertheless, this seems to be due to the dynamical evolution rather than due to an instability of the deflagration, since the detonation configuration is reached without going through a deflagration configuration. According to our results, the instability of the deflagration is unrelated to the existence of detonation 
solutions. Although the instabilities become important for large amounts of supercooling and low friction, detonations already exist for more moderate values of these parameters, where deflagrations are still stable.

Regarding possible instabilities, the results of Ref. [27] were the following. The wall configuration was found to be unstable only for strong deflagrations and for detonation solutions close to the Jouguet point. In contrast, if given as an initial condition, the weak deflagration remains as such, indicating stability. This seeming contradiction with the instability of deflagrations found in the present paper and in Ref. [14] has a simple explanation. Due to calculation convenience, the amounts of time considered in [27] (as well as the distance between bubbles) were much less than those in an actual cosmological phase transition. This is a general problem of lattice calculations. A similar calculation was carried out recently [36]. In this case, the available time did not even allow the walls to reach the stationary state before they collided. As we have seen, the time needed for the instability to become dynamically relevant is much longer than the time it takes to reach the stationary state. Nevertheless, the duration of the phase transition is still much longer.

\section{A PHYSICAL MODEL}

The strength of the phase transition [29] depends on the separation between the two minima of the free energy, $\Delta \phi=\phi_{+}-\phi_{-}$, and is usually characterized by the value of $\Delta \phi / T_{c}$ (for instance, in the limit $\Delta \phi \rightarrow 0$ one gets a second order phase transition). However, the phase transition dynamics does not depend on this single parameter alone. The wall velocity depends essentially on three parameters. These are the amount of supercooling (which determines the pressure difference), the latent heat (which reheats the plasma slowing down the wall), and the friction coefficient. As can be seen, e.g., in Eq. (133), these parameters are relevant for the dynamics of the instabilities as well. The ratio $T_{N} / T_{c}$ determines the value of $v_{c}$ and, hence, of $\beta$. The ratio $L / w_{+}$gives the parameter $\bar{L}$. Finally, the friction coefficient determines the wall velocity $v_{w}$.

Unfortunately, these parameters do not have a simple relation in general ${ }^{14}$. For a given model, both the released energy and the amount of supercooling increase with the strength of the phase transition. Indeed, a higher value of $\Delta \phi$ implies a higher discontinuity of the energy density (i.e., a higher $L$ ) as well as a wider and higher barrier between minima. The latter causes the system to stay longer in the metastable minimum, i.e., a lower temperature will be reached before bubble nucleation effectively begins. A strong supercooling causes a large pressure difference between phases and, thus, favors a high wall velocity. In contrast, a large release of latent heat causes reheating and slows the wall down. Besides, the wall velocity depends on the friction coefficient. This parameter is quite difficult to calculate, model-dependent, and is the main source of uncertainty for the wall velocity ${ }^{15}$. Therefore, it is not easy to ascertain, without a detailed calculation, whether the wall velocity will be

${ }^{14}$ While the latent heat can be directly computed from the free energy density $\mathcal{F}(\phi, T)$, the calculation of $T_{N}$ involves, first, calculating the nucleation rate (using thermal instantons [30]) and, then, considering the dynamics of the phase transition to compute the number of bubbles nucleated in a causal volume [18].

${ }^{15}$ In general, it depends on the couplings of the particles with the field $\phi$ (the stronger the coupling, the higher the friction). However, it also depends on the particles interactions which determine the diffusion of particle densities near the wall [10]. 
above $v_{\text {crit }}$ or not.

We shall address elsewhere such a detailed study of specific models. Here we wish to discuss in general the possibility that the deflagration becomes unstable in physical models, as well as some possible cosmological implications of the instability. For that aim, we shall consider the case of the electroweak phase transition, which may be quite different for different extensions of the Standard Model.

\section{A. The electroweak phase transition}

In Ref. [14], the electroweak phase transition was considered for the minimal Standard Model (SM), with a Higgs mass $m_{H}=40 \mathrm{GeV}$ in order to obtain a first-order phase transition which is strong enough to fulfil the requirement of electroweak baryogenesis $\left(\Delta \phi / T_{c} \gtrsim 1\right)$. Still, the phase transition for such a model is relatively weak and has a small amount of supercooling. The critical velocity was found to be bounded by 0.07 , whereas microscopic calculations gave $v_{w} \gtrsim 0.1[9]$. It was thus concluded that the propagation of the phase transition front as a deflagration is stable.

For the actual value of the Higgs mass, the SM electroweak phase transition is just a smooth crossover. Nevertheless, many extensions of the SM have been considered in the literature. In particular, the Minimal Supersymmetric Standard Model (MSSM) has been extensively investigated in relation with electroweak baryogenesis (see, e.g., [37]). Moreover, it is well known that extra scalar singlets may cause an extremely strong phase transition (see, e.g., [38]). Thus, depending on the model (and on the model parameters) the electroweak bubble may grow either as a deflagration or as a detonation (see, e.g., [39]), or it may even run away [24]. The former possibility favors baryogenesis, whereas the latter two favor GW generation.

In Fig. 13 we show the values of $L$ and $T_{N}$ for some extensions of the Standard Model. We only considered phase transitions with $\Delta \phi / T>1$. Weaker phase transitions give less supercooling and smaller latent heat (in the limit of a second order phase transition we have $T_{N}=T_{c}$ and $L=0$ ). Thus, the models considered in Fig. 13 give values of the latent heat in the range $\bar{L} \sim 10^{-2}-10^{-1}$. Higher values of $\bar{L}$ may be possible in other models (the physical bound is $\bar{L}<1$ ). On the other hand, it may be inferred from Fig. 13 that a very strong supercooling is hard to achieve in a physical model. Indeed, the two lower dots (those around $T_{N} / T_{c}=0.7$ ) were obtained for phase transitions which are already extremely strong (a slightly stronger phase transition would remain stuck in the false vacuum, causing an inflationary era). The numerical examples considered in Sec. V roughly spanned this region of parameter space.

As we have seen, the possible instabilities need time to grow. The initial bubble radius at nucleation, which is related to the size scale $d$, is in general $R_{i} \sim 10 / T$. On the other hand, the instabilities become dynamically important for bubble sizes larger than $R_{b}^{\text {inst }} \sim(4 / \bar{L})^{2} d$, which is thus in the range $\sim 10^{4} / T-10^{6} / T$. The average bubble size by the end of the phase transition is a fraction of the Hubble size $H^{-1}$. The exact value depends on the whole dynamics of the phase transition, and is not easy to estimate without a complete numerical calculation. Numerical results (see, e.g., [5, 41]) give values which range from $R_{b} \sim H^{-1}$ for very strong phase transitions to $R_{b} \sim 10^{-5} H^{-1}$ or smaller for weak phase transitions. At the electroweak scale we have $H^{-1} \sim M_{P} / T^{2}$, with $T \sim 100 \mathrm{GeV}$ and $M_{P} \sim 10^{19} \mathrm{GeV}$. Hence, the final bubble size for the electroweak phase transition will be in the range $R_{f} \sim 10^{12} / T-10^{17} / T$. Thus, this example confirms the general hierarchy 


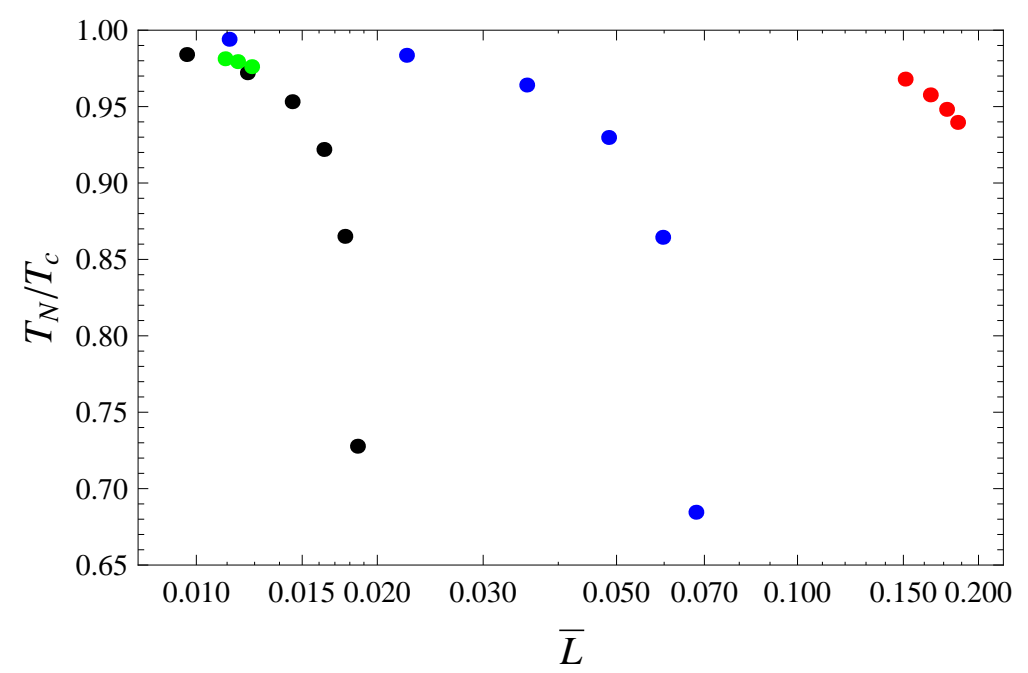

FIG. 13: The space of the parameters $\bar{L}$ and $T_{N} / T_{c}$ for the electroweak phase transition, for some extensions of the Standard Model (taken from Ref. [7]). Black dots correspond to extra scalar singlets, with $g_{s}=12$ degrees of freedom. The strength of the phase transition increases with the coupling of the scalars to the Higgs, $h_{s}$. Thus, a higher $h_{s}$ implies a larger latent heat as well as a smaller $T_{N}$. The values of $h_{s}$ range from 0.7 to 1.2 and are equally spaced. Blue dots correspond to the same extension, with less singlets, $g_{s}=2$. In this case we have $1.4 \leq h_{s} \leq 1.9$. Red dots corresponds to an extension with heavy fermions and bosons [40]. The coupling of the fermions to the Higgs is in the range $2.2 \leq h_{f} \leq 2.8$ (the strength of the phase transition increases with $h_{f}$ ). Green dots corrrespond to the MSSM in the light stop scenario, for stop masses $m_{\text {stop }}=132,136$, and $140 \mathrm{GeV}$ (from right to left).

$R_{i} \ll R_{b}^{\text {inst }} \ll R_{f}$ obtained in the previous section. The most important perturbations will be those with $\lambda \sim 4 d / \bar{L} \sim 10-10^{2} d \gtrsim R_{i}$.

\section{B. Baryogenesis and gravitational waves}

This phase transition may have several cosmological consequences, most of them depending on the dynamics of moving walls. In principle, the hydrodynamic instability may affect any of the cosmological remnants. For instance, the generation of magnetic fields due to instabilities of the bubble walls was considered in Ref. [42]. Here we wish to discuss the generation of gravitational waves (GW) and of the baryon asymmetry of the universe (BAU), which require quite different values of the wall velocity.

A successful electroweak baryogenesis requires $\Delta \phi / T_{c} \gtrsim 1$, so that baryon number violating processes (sphalerons) are turned off in the broken-symmetry phase, in order to avoid the washout of the generated BAU. Regarding the wall velocity, on the one hand, it should not be too large, so that sphalerons have enough time to generate baryons in the symmetric phase (sourced by $C P$-violating interactions of the wall with the particles of the plasma). On the other hand, the wall velocity should not be too small either, in order to avoid that sphalerons in the symmetric phase have enough time to reach the equilibrium and wash out the generated BAU. All in all, a relatively small wall velocity $\left(v_{w} \sim 10^{-2}-10^{-1}\right)$ is needed. As a consequence, baryogenesis is favored for relatively weak phase transitions, which may 
give such small wall velocities ${ }^{16}$. Weak phase transitions will generally have little supercooling and, consequently, small values of $v_{\text {crit }}$ as well. Therefore, the presence of hydrodynamic instabilities will depend on details of the specific model.

To see the effect of these potential instabilities on electroweak baryogenesis, let us assume that $v_{w}$ is below the critical velocity $v_{\text {crit }}$. As we have seen, for a weak phase transition, the instability will become dynamically important when the bubble reaches a size $R_{b}^{\text {inst }} \gtrsim 10^{6} / T$. After that moment, the growth of the bubble may be of dendritic type [45]. One expects that the motion of the wall will become too quick to successfully generate baryons [46, 47]. We may thus assume that baryogenesis stops as soon as bubbles reach the size $R_{b}^{\text {inst }}$. Since the final bubble size is $R_{f} \sim 10^{6} R_{b}^{\text {inst }}$, we see that the resulting BAU will be strongly suppressed with respect to a stable wall ${ }^{17}$. We see that an accurate determination of the wall velocity becomes crucial since electroweak baryogenesis may be completely spoiled if $v_{w}<v_{\text {crit }}$.

On the other hand, we have seen that, once shock fronts meet and reheat the plasma, the motion of phase transition interfaces as stable deflagrations may be reestablished. Depending on the friction and latent heat, the value of the wall velocity during this phase-equilibrium stage may or may not be appropriate for baryogenesis [4, 5, 48]. In case it is, bubble walls will generate baryons during the last stages of the phase transition. It is important to notice that a significant fraction of space may be spanned by the walls during this stage.

Generating gravitational waves of sizeable intensity generally requires quite higher velocities $\left(v_{w}>c_{s}\right)$ in order to generate a strong disturbance of the plasma (through bubble collisions and turbulence). Hence, the instability of the deflagration is preferable, as it accelerates the wall motion. In fact, gravitational waves of sizeable amplitude seem to be possible only in models with large amounts of supercooling (e.g., the lower dots in Fig. 13), which give detonations with high velocities [7]. Such models may also allow deflagrations with velocities $v_{w}$ close to $c_{s}$ or higher. In general, these models will give $v_{\text {crit }}$ also close to $c_{s}$. According to Fig. 11, in this case the deflagration may have instabilities on all wavelengths (notice the divergence of $k_{c}$ at $v_{w}=c_{s}$ ). This opens the possibility of a new mechanism of GW generation, which may compete with the collisions of detonations, even for weaker phase transitions.

The evolution of the system beyond the linear regime is difficult to guess. Furthermore, it will be characterized by turbulent motions of the fluid, which make the treatment more involved. The results of a simple geometrical model (described by an equation which depends only on the local geometry of the interface) suggest that the growth may be of dendritic type [45]. This means that "fingers" grow out of the wall and then split into new fingers.

A spherically symmetric bubble cannot generate gravitational radiation. As a consequence, the usual mechanisms (bubble collisions and turbulence) rely on the collision of bubble walls, once bubbles have grown up to there final size. The corrugation instability, in contrast, deforms the walls and stirs the fluid as soon as the bubble reaches the size $R_{b}^{\text {inst }} \sim(4 / \bar{L})^{2} R_{i}$, when bubbles are still much smaller than the final mean size. Therefore, the GW spectrum will be quite different. The characteristic wavelength of the gravitational radiation is given by the stirring scale. For the usual mechanisms, this is roughly the bubble size scale $R_{b}$, which is determined by the mean average separation between nucleation

\footnotetext{
$\overline{16}$ The fact that the incoming flow velocity $\left|v_{+}\right|$is smaller than $v_{w}$ may increase the upper bound 43$]$. Moreover, the possibility of electroweak baryogenesis with detonations has been recently discussed [4].

${ }^{17}$ In this argument we have used the rough approximation $R_{b}^{\text {inst }} / d \sim(4 / \bar{L})^{2}$ for $v_{w}<v_{\text {crit }}$. Taking into account the factor $1 /\left(1-v_{w}^{2} / v_{\text {crit }}^{2}\right)$ will not change the conclusion, unless $v_{w}$ is very close to $v_{\text {crit }}$.
} 
points. In the case of unstable growth, the relevant scale (or scales) will be smaller.

Initially, the source of turbulence will be the unstable corrugations of the wall (accompanied by perturbations of the fluid). Thus, the initial stirring length scale is that of the most relevant unstable mode, $\lambda_{\text {inst }} \sim 2 / k_{c} \sim 4 R_{i} / \bar{L}$. These perturbations then grow in size and amplitude. In the case of dendritic growth, a new length scale may arise, namely, the length of the fingers. In any case, after a certain time the turbulent fluid will "see" also the nominal radius of the bubbles $R_{b} \sim R_{b}^{\text {inst }}$. This gives another stirring scale. The bubble spacing $R_{f}$ may also play a role in the turbulence spectrum. As we have seen, both $\lambda_{\text {inst }}$ and $R_{b}^{\text {inst }}$ are much smaller than $R_{f}$. For the usual mechanisms, the (redshifted) peak of the spectrum is around the miliHertz (corresponding to $R_{f} \sim 10^{-2} H^{-1}$ ). For the unstable growing, the GW spectrum may have several peaks, some of them at frequencies much higher than that.

\section{CONCLUSIONS}

The possibility that an observable background of gravitational waves was produced at the electroweak phase transition has motivated in the last years a renewed interest in the hydrodynamics associated to the propagation of phase transition fronts. It is well known that, while electroweak baryogenesis requires weak deflagrations with rather small interface velocities, $v_{w} \lesssim 0.1$, GW generation is favored by detonations or runaway solutions with ultra-relativistic velocities. Thus, the various extensions of the SM give quite different results, depending on the values of three relevant parameters, namely, the amount of supercooling, the latent heat, and the friction. In particular, small supercooling, large friction, and large latent heat will give in general small wall velocities, favoring baryogenesis. The instability of deflagrations may alter completely this picture.

In this work, we have studied the hydrodynamic stability of deflagrations. We have calculated the linear instability under corrugation of the wall as a function of the relevant parameters, we have analyzed the dynamical relevance of the instabilities, and we have discussed the implications for the electroweak phase transition and its cosmological consequences.

The instability of deflagration phase-transition fronts was previously considered in Ref. [14]. The treatment of that work improved significantly upon preceding analysis, by taking into account the perturbations of the force which drives the wall motion. This is an important aspect, since the pressure difference between phases is very sensitive to temperature variations. Unfortunately, some simplifications used for the driving force constrain the application of those results. Our approach improved several aspects of the calculation of Ref. [14]. In the first place, we have derived the equation for the perturbations of the wall directly from the field equation (2), taking into account independent perturbations of the fluid variables on either side of the wall. This is the main difference with the treatment of Ref. [14]. Its quantitative effect increases with the wall velocity. We have also performed a more exhaustive search of instabilities. In particular, we have looked for complex solutions of the equation for the exponential growth rate $\Omega$. In the case of a classical burning gas [12], the unstable modes have $\operatorname{Im}(\Omega)=0$. Thus, the disturbances are not propagated but are only amplified. This feature was also found (numerically) in the work of Link [13]. We investigated analytically as well as numerically this possibility for the case of a phase transition front. The result is that, indeed, we have $\operatorname{Im}(\Omega)=0$ for $\operatorname{Re}(\Omega)>0$.

For small velocities and small supercooling, our results are qualitatively similar to those of Refs. [13] and [14]. However, we have found a range of marginally unstable wavenumbers, which was not noticed in previous works. Outside this interval we have exponential (either 
growing or decaying) behavior. This wavenumber gap arises as a discontinuity at $\Omega=0$, and is due to the fact that the special mode $q_{1}(\Omega)$ jumps from one side of the wall to the other as $\Omega$ changes sign. Studying the stability in this range would require to go beyond linear perturbations. Unfortunately, the numerical analysis of Ref. [15] did not explore regions of parameters where our results would differ from those of Ref. [14]. Moreover, a numerical investigation of the parameter region where linear perturbation theory predicts instabilities is still lacking.

The general behavior of the linear stability is essentially the following. Below a critical velocity $v_{\text {crit }}$, perturbations on wavenumbers $k$ smaller than a value $k_{c}$ are exponentially unstable. In general, we have $k_{c} \lesssim \bar{L} / d$, and $\Omega \lesssim v_{w} \bar{L} k_{c}$. The critical velocity depends strongly on the amount of supercooling. For small supercooling, we have $v_{\text {crit }} \simeq \sqrt{1-T_{+} / T_{c}}$, in agreement with Ref. [14]. However, as we increase the amount of supercooling $v_{\text {crit }}$ quickly departs from this simple behavior. Even taking into account the reheating effect $T_{+}>T_{N}$, the critical velocity soon approaches the speed of sound, which means that any subsonic velocity becomes unstable. Furthermore, in this case, those velocities which are closer to the speed of sound have a larger range of unstable wavenumbers and higher growth rates. This result is in disagreement with Ref. [14], according to which weak deflagrations are always stable in the limit $v_{w} \rightarrow c_{s}$. The discrepancy is due to our more realistic treatment of the equation for the interface.

We have briefly discussed supersonic deflagrations. The case of supersonic Jouguet deflagrations turns out to be considerably more involved, and shall be addressed elsewhere. Regarding strong deflagrations, we have checked, for the case of planar relativistic phasetransition fronts, that these are trivially unstable, by showing explicitly that the whole family of strong deflagrations (sketched in Fig. 3, left panel) is not evolutionary.

We have also studied the dynamical importance of the instabilities. Thus, we have improved the discussions of Refs. [13, 34], and we have established a hierarchy of time and length scales for the growth of bubbles and instabilities.

We also discussed briefly a physical model, namely, the electroweak phase transition, and considered two of its possible outcomes, namely, the BAU of the universe and a stochastic background of gravitational waves. In general, for a cosmological phase transition, the instabilities have ample time to develop, provided that $v_{w}<v_{\text {crit }}$. This may be a serious problem for electroweak baryogenesis and deserves further investigation for specific models. On the other hand, the deflagration instability favors the production of gravitational waves, by accelerating and deforming the walls almost from the beginning of bubble growth. However, to estimate the GW spectrum would require to go beyond the linear stability analysis.

\section{Acknowledgements}

This work was supported by Universidad Nacional de Mar del Plata, Argentina, grant EXA $607 / 12$.

[1] For a review, see D. Grasso and H. R. Rubinstein, Phys. Rept. 348, 163 (2001) arXiv:astro-ph/0009061. 
[2] A. Vilenkin and E.P.S. Shellard, Cosmic Strings and Other Topological Defects (Cambridge University Press, Cambridge, England, 1994); A. Vilenkin, Phys. Rept. 121, 263 (1985).

[3] E. Witten, Phys. Rev. D 30, 272 (1984); G. M. Fuller, G. J. Mathews and C. R. Alcock, Phys. Rev. D 37, 1380 (1988); J. H. Applegate and C. J. Hogan, Phys. Rev. D 31, 3037 (1985); H. Kurki-Suonio, Phys. Rev. D 37, 2104 (1988); J. Ignatius, K. Kajantie, H. Kurki-Suonio and M. Laine, Phys. Rev. D 50, 3738 (1994) arXiv:hep-ph/9405336.

[4] A. F. Heckler, Phys. Rev. D 51 (1995) 405 arXiv:astro-ph/9407064;

[5] A. Megevand and F. Astorga, Phys. Rev. D 71, 023502 (2005) [hep-ph/0409321].

[6] See, e.g., A. Kosowsky and M. S. Turner, Phys. Rev. D 47, 4372 (1993); M. Kamionkowski, A. Kosowsky and M. S. Turner, Phys. Rev. D 49, 2837 (1994); A. Kosowsky, A. Mack and T. Kahniashvili, Phys. Rev. D 66, 024030 (2002); A. D. Dolgov, D. Grasso and A. Nicolis, Phys. Rev. D 66, 103505 (2002); C. Caprini and R. Durrer, Phys. Rev. D 74, 063521 (2006); C. Caprini, R. Durrer and G. Servant, Phys. Rev. D 77, 124015 (2008) arXiv:0711.2593 [astro-ph]]; R. Apreda, M. Maggiore, A. Nicolis and A. Riotto, Nucl. Phys. B 631, 342 (2002); A. Nicolis, Class. Quant. Grav. 21, L27 (2004); C. Grojean and G. Servant, Phys. Rev. D 75, 043507 (2007); S. J. Huber and T. Konstandin, JCAP 0809, 022 (2008) arXiv:0806.1828 [hep-ph]]; S. J. Huber and T. Konstandin, JCAP 0805, 017 (2008) arXiv:0709.2091 [hep-ph]]. A. Megevand, Phys. Rev. D 78 (2008) 084003 arXiv:0804.0391 [astro-ph]]; J. Kehayias and S. Profumo, JCAP 1003, 003 (2010) arXiv:0911.0687 [hep-ph]].

[7] L. Leitao, A. Megevand and A. D. Sanchez, JCAP 1210, 024 (2012) arXiv:1205.3070 [astroph.CO]].

[8] For reviews, see A. G. Cohen, D. B. Kaplan and A. E. Nelson, Ann. Rev. Nucl. Part. Sci. 43, 27 (1993) arXiv:hep-ph/9302210; A. Riotto and M. Trodden, Ann. Rev. Nucl. Part. Sci. 49, 35 (1999) arXiv:hep-ph/9901362.

[9] M. Dine, R. G. Leigh, P. Y. Huet, A. D. Linde and D. A. Linde, Phys. Rev. D 46, 550 (1992) arXiv:hep-ph/9203203; B. H. Liu, L. D. McLerran and N. Turok, Phys. Rev. D 46, 2668 (1992).

[10] See, e.g., N. Turok, Phys. Rev. Lett. 68, 1803 (1992); S. Y. Khlebnikov, Phys. Rev. D 46, 3223 (1992); P. Arnold, Phys. Rev. D 48, 1539 (1993) arXiv:hep-ph/9302258; G. D. Moore and T. Prokopec, Phys. Rev. D 52, 7182 (1995) arXiv:hep-ph/9506475; Phys. Rev. Lett. 75, 777 (1995) arXiv:hep-ph/9503296]; P. John and M. G. Schmidt, Nucl. Phys. B 598, 291 (2001) [Erratum-ibid. B 648, 449 (2003)]; G. D. Moore, JHEP 0003, 006 (2000).

[11] See, e.g., M. Gyulassy, K. Kajantie, H. Kurki-Suonio and L. D. McLerran, Nucl. Phys. B 237, 477 (1984); H. Kurki-Suonio, Nucl. Phys. B 255, 231 (1985); K. Kajantie and H. Kurki-Suonio, Phys. Rev. D 34, 1719 (1986); K. Enqvist, J. Ignatius, K. Kajantie and K. Rummukainen, Phys. Rev. D 45, 3415 (1992).

[12] L. D. Landau and E. M. Lifshitz, Fluid Mechanics (Pergamon Press, New York, 1989).

[13] B. Link, Phys. Rev. Lett. 68, 2425 (1992).

[14] P. Y. Huet, K. Kajantie, R. G. Leigh, B. H. Liu and L. D. McLerran, Phys. Rev. D 48, 2477 (1993) arXiv:hep-ph/9212224].

[15] P. C. Fragile and P. Anninos, Phys. Rev. D 67, 103010 (2003) gr-qc/0303018.

[16] M. Abney, Phys. Rev. D 49, 1777 (1994) astro-ph/9305021.

[17] L. Rezzolla, Phys. Rev. D 54, 1345 (1996) astro-ph/9605033.

[18] A. H. Guth and E. J. Weinberg, Phys. Rev. D 23, 876 (1981).

[19] G. W. Anderson and L. J. Hall, Phys. Rev. D 45, 2685 (1992).

[20] A. Mégevand, Int. J. Mod. Phys. D 9, 733 (2000) hep-ph/0006177]. 
[21] J. R. Espinosa, T. Konstandin, J. M. No and G. Servant, JCAP 1006, 028 (2010) arXiv:1004.4187 [hep-ph]];

[22] S. J. Huber and M. Sopena, arXiv:1302.1044 [hep-ph].

[23] A. Megevand, JCAP 1307, 045 (2013) arXiv:1303.4233 [astro-ph.CO]].

[24] D. Bodeker and G. D. Moore, JCAP 0905, 009 (2009) arXiv:0903.4099 [hep-ph]].

[25] A. Megevand and A. D. Sanchez, Nucl. Phys. B 865, 217 (2012) arXiv:1206.2339 [astroph.CO]].

[26] L. Leitao and A. Megevand, Nucl. Phys. B 844, 450 (2011) arXiv:1010.2134 [astro-ph.CO]].

[27] J. Ignatius, K. Kajantie, H. Kurki-Suonio and M. Laine, Phys. Rev. D 49, 3854 (1994);

H. Kurki-Suonio and M. Laine, Phys. Rev. D 51, 5431 (1995) arXiv:hep-ph/9501216;

H. Kurki-Suonio and M. Laine, Phys. Rev. D 54, 7163 (1996) hep-ph/9512202].

[28] A. Megevand and A. D. Sanchez, Nucl. Phys. B 820, 47 (2009) arXiv:0904.1753 [hep-ph]].

[29] M. Quiros, arXiv:hep-ph/9901312.

[30] A. D. Linde, Nucl. Phys. B 216, 421 (1983) [Erratum-ibid. B 223, 544 (1983)]; Phys. Lett. B 100, 37 (1981).

[31] A. Megevand and F. A. Membiela, arXiv:1402.5791 [astro-ph.CO].

[32] T. Konstandin and J. M. No, JCAP 1102, 008 (2011) [arXiv:1011.3735 [hep-ph]].

[33] P. J. Steinhardt, Phys. Rev. D 25, 2074 (1982).

[34] K. Kajantie, Phys. Lett. B 285, 331 (1992).

[35] A. Megevand, Phys. Rev. D 69, 103521 (2004) hep-ph/0312305.

[36] M. Hindmarsh, S. J. Huber, K. Rummukainen and D. J. Weir, arXiv:1304.2433 [hep-ph].

[37] M. S. Carena, M. Quiros and C. E. M. Wagner, Nucl. Phys. B 524, 3 (1998) arXiv:hep-ph/9710401. J. A. Casas, J. R. Espinosa, M. Quiros and A. Riotto, Nucl. Phys. B 436, 3 (1995) [Erratum-ibid. B 439, 466 (1995)] arXiv:hep-ph/9407389 M. S. Carena, M. Quiros and C. E. M. Wagner, Phys. Lett. B 380, 81 (1996) arXiv:hep-ph/9603420. M. S. Carena and C. E. M. Wagner, Nucl. Phys. B 452, 45 (1995) arXiv:hep-ph/9408253; J. R. Espinosa, Nucl. Phys. B 475, 273 (1996) arXiv:hep-ph/9604320]; J. E. Bagnasco and M. Dine, Phys. Lett. B 303, 308 (1993) arXiv:hep-ph/9212288; P. Arnold and O. Espinosa, Phys. Rev. D 47, 3546 (1993) [Erratum-ibid. D 50, 6662 (1994)] arXiv:hep-ph/9212235]; Z. Fodor and A. Hebecker, Nucl. Phys. B 432, 127 (1994) arXiv:hep-ph/9403219].

[38] M. Dine, P. Huet, R. L. Singleton and L. Susskind, Phys. Lett. B 257, 351 (1991); M. Dine, P. Huet and R. L. . Singleton, Nucl. Phys. B 375, 625 (1992); J. Choi and R. R. Volkas, Phys. Lett. B 317, 385 (1993) arXiv:hep-ph/9308234; S. W. Ham, Y. S. Jeong and S. K. Oh, J. Phys. G 31, 857 (2005) arXiv:hep-ph/0411352; J. R. Espinosa and M. Quiros, Phys. Lett. B 305, 98 (1993) arXiv:hep-ph/9301285]; A. Ahriche, Phys. Rev. D 75, 083522 (2007) arXiv:hep-ph/0701192 ; S. Profumo, M. J. Ramsey-Musolf and G. Shaughnessy, JHEP 0708, 010 (2007) [arXiv:0705.2425 [hep-ph]]; A. Ashoorioon and T. Konstandin, JHEP 0907, 086 (2009) arXiv:0904.0353 [hep-ph]]. J. R. Espinosa and M. Quiros, Phys. Rev. D 76, 076004 (2007) arXiv:hep-ph/0701145].

[39] A. Megevand and A. D. Sanchez, Nucl. Phys. B 825, 151 (2010) arXiv:0908.3663 [hep-ph]].

[40] M. S. Carena, A. Megevand, M. Quiros and C. E. M. Wagner, Nucl. Phys. B 716, 319 (2005) arXiv:hep-ph/0410352.

[41] A. Megevand and A. D. Sanchez, Phys. Rev. D 77, 063519 (2008) [arXiv:0712.1031 [hep-ph]].

[42] G. Sigl, A. V. Olinto and K. Jedamzik, Phys. Rev. D 55, 4582 (1997) astro-ph/9610201.

[43] J. M. No, Phys. Rev. D 84, 124025 (2011) [arXiv:1103.2159 [hep-ph]].

[44] C. Caprini and J. M. No, JCAP 1201, 031 (2012) [arXiv:1111.1726 [hep-ph]]. 
[45] K. Freese and F. C. Adams, Phys. Rev. D 41, 2449 (1990).

[46] M. Kamionkowski and K. Freese, Phys. Rev. Lett. 69, 2743 (1992) hep-ph/9208202].

[47] M. Abney, Phys. Rev. D 55, 582 (1997) hep-ph/9606476].

[48] A. Megevand, Phys. Rev. D 64, 027303 (2001) hep-ph/0011019. 